\title{
Unifying Equivalences for Timed Transition Systems*
}

\author{
Irina Virbitskaite ${ }^{1,2}$, Nataliya Gribovskaya ${ }^{1}$ and Eike Best ${ }^{3}$ \\ 1 A.P. Ershov Institute of Informatics Systems, \\ SB RAS, Novosibirsk, Russia \\ virb@iis.nsk.su \\ 2 Novosibirsk State University, Novosibirsk, Russia \\ 3 Carl von Ossietzky University of Oldenburg, \\ Oldenburg, Germany
}

\begin{abstract}
Timed transition systems are a widely studied model for real-time systems. The intention of the paper is to show how several categorical (open maps, path-bisimilarity and coalgebraic) approaches to an abstract characterization of bisimulation relate to each other and to the numerous suggested behavioral equivalences of linear time - branching time spectrum, in the setting of timed transition systems.
\end{abstract}

\section{Introduction}

For the purpose of specification and verification of the behavior of systems, it is necessary to provide a number of suitable equivalence notions in order to be able to choose the simplest possible view of the system. Over the past several years, a variety of equivalences have been promoted, and the relationships between them have been understood quite well (see, for example, [25, 6]).

In order to unify and clarify apparent differences between the extensive amount of research within the field of behavioral equivalences, several category-theoretic approaches to the matter have appeared. Two of them were initiated by Joyal, Nielsen, and Winskel in [14 where they have proposed abstract ways of capturing the notion of behavioral equivalence through open maps based bisimilarity and its logical counterpart — path bisimilarity. As shown in [5, 14, 20, bisimilarity induced by open maps makes possible a uniform definition of the numerous suggested behavioral equivalences (e.g., trace and testing equivalences, bisimulation, barbed and weak bisimulations, strong history preserving bisimulation, etc.) across a wide range of models for concurrency (e.g., transition systems, event structures, Petri nets, higher dimensional automata, etc.).

Another way to provide categorical characterizations is to adopt the coalgebraic approach which has both a field of its own interest presenting a deep mathematical foundation and a growing field of applications and interactions with various other approaches such as reactive and interactive system theory, object-oriented and concurrent programming, formal system specification, modal logic, etc. During the last years, it is becoming increasingly clear that a great variety of state-based dynamical systems, like transition systems, automata, process calculi and class-based systems can be captured uniformly as coalgebras. There is also a coalgebraic notion of bisimulation, the research in this area has been initiated by Aczel and Mendler 1. Since then several papers have emerged in the literature (see [12, 16, 22, 18, 21, among others). One of the basic strands of the research is concerned with a coalgebraic rendering of various behavioral equivalences in the linear time - branching time spectrum.

*This work is supported in part by DFG (grant No 436 RUS 113/1002/01) 
Over the last two decades, much of the theory of observational equivalences of models has been lifted to real-time settings (see [2, 3, 4, 26, 10, 24] among others). The situation is less settled in the case of categorical unification of time-sensitive equivalences. In [11] and [27, the open maps based approach has been applied to provide an abstract characterization of bisimulation on timed transition systems and of partial order based equivalences on timed event structures, respectively. The categorical framework of open maps has been used in 9] to prove that timed delay equivalence is indeed an equivalence relation in the setting of timed transition systems with invariants. The paper [15] has given a coalgebraic formulation of timed processes and their operational semantics, where time is modelled by a monoid called a time domain, and processes are modelled by timed transition systems, which amount to coalgebras for an evolution comonad generated by the time domain.

The contribution of the paper is to show how several categorical (open maps, pathbisimilarity and coalgebraic) approaches to an abstract characterization of bisimulation relate to each other and to the numerous suggested behavioral equivalences in the setting of timed transition systems. Such an approach makes it possible to develop a metatheory designed for unified definition and study of behavioral equivalences in linear time - branching time spectrum of timed semantics.

The rest of the paper is organized as follows. The basic notions and notations related to timed transition systems and their behavior are introduced in section 2. In the next section, we define a number of behavioral equivalences for timed transition systems. Different categories of timed transition systems are introduced and open maps based characterizations of the behavioral equivalences are given in section 4 . In section 5 , we show how the equivalences under consideration can be captured by another category-theoretic bisimulation - path-bisimulation. In section 6 , a coalgebraic formulation of the equivalences is treated.

\section{Timed Transition Systems}

In this section, we define some basic notions concerning the structure and behavior of timed transition systems [11].

Before doing so, it will be convenient to introduce some auxiliary notions and notations. Let $\mathbf{R}$ be the set of non-negative reals. Also, let $\Sigma$ be a finite alphabet of actions without the silent action $\tau$, and $\Sigma_{\tau}=\Sigma \cup\{\tau\}$. A timed word over $\Sigma$ (resp. $\Sigma_{\tau}$ ) is a finite sequence of pairs $\alpha=\left(\sigma_{1}, d_{1}\right) \ldots\left(\sigma_{n}, d_{n}\right)$ such that $\sigma_{i} \in \Sigma$ (resp. $\left.\sigma_{i} \in \Sigma_{\tau}\right), d_{i} \in \mathbf{R}$, for all $1 \leq i \leq n$, and $d_{i}<d_{i+1}$ for all $1 \leq i<n$. A pair $\left(\sigma_{i}, d_{i}\right)$ represents an occurrence of an action $\sigma_{i}$ at time $d_{i}$ relative to the starting time $(0)$ of the execution. Let $\varepsilon$ denote the empty timed word. We consider a finite set $V$ of clock variables. A clock valuation over $V$ is a mapping $\nu: V \rightarrow \mathbf{R}$ which assigns time values to the clock variables of a system. Define $(\nu+c)(x):=\nu(x)+c$ for all clock variables $x \in V$ and constants $c \in \mathbf{R}$. For a subset $\lambda$ of clock variables, we shall write $\nu[\lambda \rightarrow 0](x):=0$, if $x \in \lambda$, and $\nu[\lambda \rightarrow 0](x):=\nu(x)$, otherwise. Given a set $V$, we define the set $\Delta(V)$ of clock constraints by the following grammar: $\delta::=c \# x|x+c \# y|$ $\delta \wedge \delta$, where $\# \in\{\leq,<, \geq,>,=\}, c$ is a real valued constant and $x, y$ are clock variables from $V$. We shall say that a clock constraint $\delta$ is satisfied by a clock valuation $\nu$ if the expression $\delta[\nu(x) / x]^{1}$ evaluates to true. A clock constraint $\delta$ defines a subset of $\mathbf{R}^{m}$ ( $m$ is the number of clock variables in $V$ ). We call the subset as the meaning of $\delta$ and denote it as $\|\delta\|_{V}$. A clock valuation $\nu$ defines a point in $\mathbf{R}^{m}$ (denoted $\|\nu\|_{V}$ ). So, the clock constraint $\delta$ is satisfied by the clock valuation $\nu$ iff $\|\nu\|_{V} \in\|\delta\|_{V}$.

\footnotetext{
${ }^{1} \delta[y / x]$ is the substitution of $y$ for $x$ in $\delta$.
} 


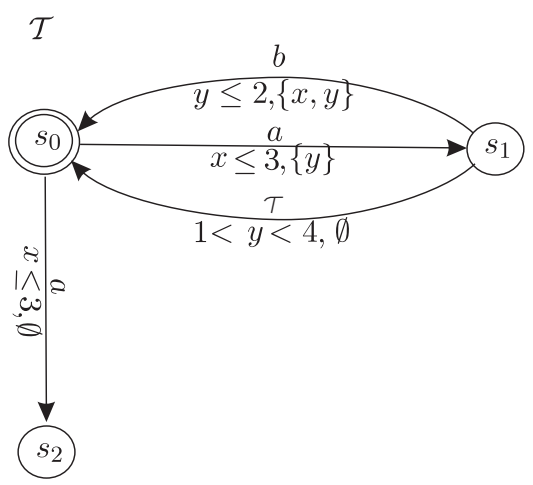

Figure 1: An example of a timed transition system over $\Sigma_{\tau}$

We are now prepared to consider the definition of timed transition systems.

Definition 1. A timed transition system $\mathcal{T}$ over an alphabet $\Sigma$ (resp. $\Sigma_{\tau}$ ) is a quintuple $\left(S, s_{0}, \Sigma, V, T\right)$ (resp. $\left(S, s_{0}, \Sigma_{\tau}, V, T\right)$ ), where $S$ is a set of states and $s_{0}$ is the initial state, $V$ is a set of clock variables, $T \subseteq S \times \Sigma \times \Delta(V) \times 2^{V} \times S$ (resp. $T \subseteq S \times \Sigma_{\tau} \times \Delta(V) \times 2^{V} \times S$ ) is a set of transitions. We shall write $s \underset{\delta, \lambda}{\stackrel{\sigma}{\rightarrow}} s^{\prime}$ to denote a transition $\left(s, \sigma, \delta, \lambda, s^{\prime}\right)$.

An example of a timed transition system over $\Sigma_{\tau}$ is depicted in Fig 1.

Define the behavior of timed transition systems.

Definition 2. Let $\mathcal{T}$ be a timed transition system over $\Sigma\left(\Sigma_{\tau}\right)$.

A configuration of $\mathcal{T}$ is a pair $\langle s, \nu\rangle$, where $s$ is a state and $\nu$ is a clock valuation. A configuration $\langle s, \nu\rangle$ of $\mathcal{T}$ is called initial iff $s$ is the initial state and $\nu$ is the constant 0 function.

$A$ run of $\mathcal{T}$ is a sequence $\gamma=\left\langle s_{0}, \nu_{0}\right\rangle \underset{d_{1}}{\stackrel{\sigma_{1}}{\rightarrow}}\left\langle s_{1}, \nu_{1}\right\rangle \ldots\left\langle s_{n-1}, \nu_{n-1}\right\rangle \underset{d_{n}}{\stackrel{\sigma_{n}}{\rightarrow}}\left\langle s_{n}, \nu_{n}\right\rangle$ such that for all $0<i \leq n$ there is a transition $s_{i-1} \underset{\delta_{i}, \lambda_{i}}{\stackrel{\sigma_{i}}{\rightarrow}} s_{i}$ such that $\left\|\nu_{i-1}+\left(d_{i}-d_{i-1}\right)\right\|_{V} \in\left\|\delta_{i}\right\|_{V}$ and $\nu_{i}=\left(\nu_{i-1}+\left(d_{i}-d_{i-1}\right)\right)\left[\lambda_{i} \rightarrow 0\right]$. Here, $\left\langle s_{0}, \nu_{0}\right\rangle$ is the initial configuration and $d_{0}$ is defined to be 0 . We will use Runs $(\mathcal{T})$ to denote the set of runs of $\mathcal{T}$. A run $\gamma$ as above is said to generate the timed word $\alpha=\left(\sigma_{1}, d_{1}\right) \ldots\left(\sigma_{n}, d_{n}\right)$. A configuration $\langle s, \nu\rangle$ of $\mathcal{T}$ is called reachable iff $\mathcal{T}$ has a run with an occurrence of $\langle s, \nu\rangle$. The set of all reachable configurations of $\mathcal{T}$ is denoted as $\operatorname{Conf}(\mathcal{T})$.

\section{Behavioral Equivalences}

In this section, we define a number of behavioral equivalences for timed transition systems.

Timed Trace Equivalence. Timed trace equivalence [2] is perhaps the first and simplest equivalence between timed transition systems that one can think of.

Definition 3. The language of a timed transition system $\mathcal{T}$ over $\Sigma$ is the set $\mathcal{L}(\mathcal{T})=\{\alpha=$ $\left.\left(\sigma_{1}, d_{1}\right) \ldots\left(\sigma_{n}, d_{n}\right) \mid\left\langle s_{0}, \nu_{0}\right\rangle \underset{d_{1}}{\stackrel{\sigma_{1}}{\rightarrow}}\left\langle s_{1}, \nu_{1}\right\rangle \ldots\left\langle s_{n-1}, \nu_{n-1}\right\rangle \underset{d_{n}}{\stackrel{\sigma_{n}}{\rightarrow}}\left\langle s_{n}, \nu_{n}\right\rangle \in \operatorname{Runs}(\mathcal{T})\right\}$.

Timed transition systems $\mathcal{T}$ and $\mathcal{T}^{\prime}$ over $\Sigma$ are called timed trace equivalent (or traceequivalent) iff $\mathcal{L}(\mathcal{T})=\mathcal{L}\left(\mathcal{T}^{\prime}\right)$. 

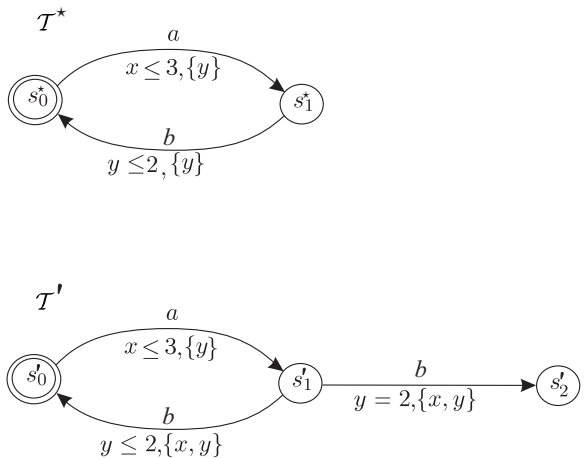

$\widehat{T}$

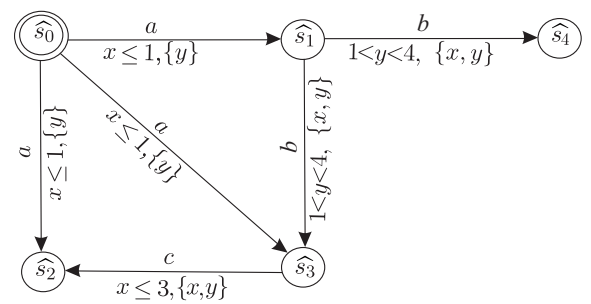

$\widetilde{\mathcal{T}}$

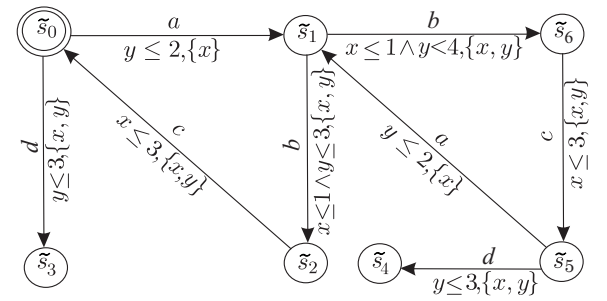

(c) (b)

(a)
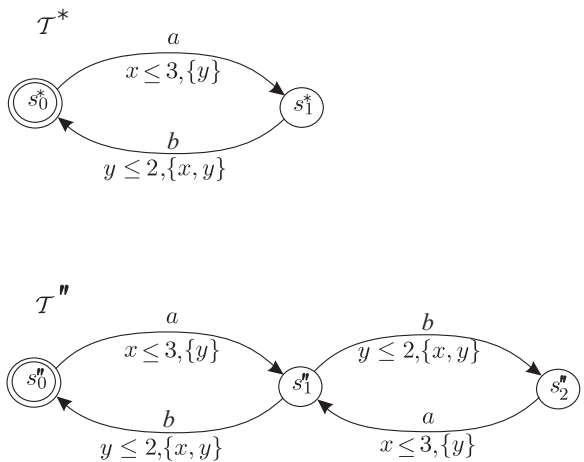

$\check{\mathcal{T}}$

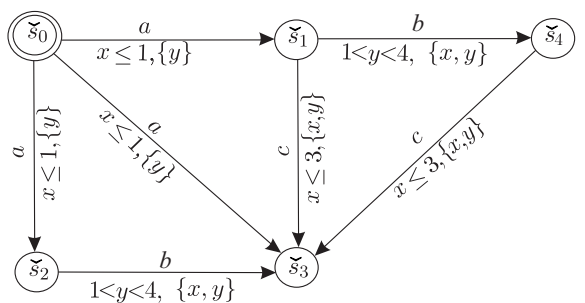

$\overline{\mathcal{T}}$

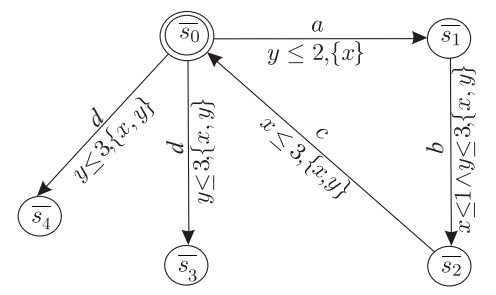

(d)

Figure 2: Examples of non-trace-equivalent $(a)$, trace- and non-test-equivalent $(b)$, test- and non-bis-equivalent $(c)$, bis-equivalent $(d)$ timed transition systems

Example 1. Consider the timed transition systems $\mathcal{T}^{\star}$ and $\mathcal{T}^{*}$ shown in Fig. 2(a). It is easy to see that $\mathcal{L}\left(\mathcal{T}^{\star}\right)=\left\{\alpha \mid \alpha \beta=\left(a, d_{1}\right)\left(b, d_{2}\right)\left(a, d_{3}\right)\left(b, d_{4}\right) \ldots\left(a, d_{2 n-1}\right)\left(b, d_{2 n}\right)(n \geq 0)\right.$, $\left.d_{2 j+1} \leq 3(0 \leq j \leq n-1), d_{2 j}-d_{2 j-1} \leq 2(1 \leq j \leq n), d_{0}=0\right\}$, and $\mathcal{L}\left(\mathcal{T}^{*}\right)=\{\alpha \mid$ $\alpha \beta=\left(a, d_{1}\right)\left(b, d_{2}\right)\left(a, d_{3}\right)\left(b, d_{4}\right) \ldots\left(a, d_{2 n-1}\right)\left(b, d_{2 n}\right)(n \geq 0), d_{2 j+1}-d_{2 j} \leq 3(0 \leq j \leq n-1)$, $\left.d_{2 j}-d_{2 j-1} \leq 2(1 \leq j \leq n), d_{0}=0\right\}$. Then, we have $\mathcal{L}\left(\mathcal{T}^{\star}\right) \subset \mathcal{L}\left(\mathcal{T}^{*}\right)$. This means that the systems $\mathcal{T}^{\star}$ and $\mathcal{T}^{*}$ are not trace-equivalent. Indeed, for example, the timed word $(a, 3)(b, 4)(a, 5)$ belongs to $\mathcal{L}\left(\mathcal{T}^{*}\right)$ but does not to $\mathcal{L}\left(\mathcal{T}^{\star}\right)$. On the other hand, the timed transition systems $\mathcal{T}^{\prime}$ and $\mathcal{T}^{\prime \prime}$ shown in Fig. 2(b) are trace-equivalent because their languages are equal to the set $\{\alpha$ $\mid \alpha \beta=\left(a, d_{1}\right)\left(b, d_{2}\right) \ldots\left(a, d_{2 k+1}\right)\left(b, d_{2 k+2}\right)(k \geq 0), d_{2 i+1}-d_{2 i} \leq 3, d_{2 i+2}-d_{2 i+1} \leq 2$ 
$\left.(i=0, \ldots, k), d_{0}=0\right\}$.

Timed Testing. Testing equivalences [19 are defined in terms of tests which processes may and must satisfy. Two processes are considered testing equivalent if there is no test that can distinguish them. A test is usually itself a process applied to a process by computing both together in parallel. A particular computation is assumed to be successful if the test reaches a designated successful state, and the process guarantees the test if every computation is successful. However, following the paper [8, we use an alternative characterization of the testing concept. In timed interleaving semantics, a test consists of a timed word and a set of actions with times at which the actions occur. A process passes this test if after every execution of the timed word, an occurrence of at least one action at time from the set is inevitably next.

Definition 4. Let $\mathcal{T}$ and $\mathcal{T}^{\prime}$ be timed transition systems over $\Sigma$. Then,

- for a timed word $\alpha=\left(\sigma_{1}, d_{1}\right) \ldots\left(\sigma_{n}, d_{n}\right)$ over $\Sigma$ and for a subset $L \subseteq(\Sigma \times \mathbf{R}), \mathcal{T}$ after $\alpha$ MUST $L$ iff for all $\langle s, \nu\rangle \in \operatorname{Conf}(\mathcal{T})$ reachable by a run of $\alpha$, there exists $(\sigma, d) \in L$ such that $\langle s, \nu\rangle \underset{d}{\stackrel{\sigma}{\rightarrow}}\left\langle s^{\prime}, \nu^{\prime}\right\rangle$, for some $\left\langle s^{\prime}, \nu^{\prime}\right\rangle \in \operatorname{Conf}(\mathcal{T})$,

- $\mathcal{T}$ and $\mathcal{T}^{\prime}$ are timed testing equivalent (or test-equivalent) iff for all timed words $\alpha=$ $\left(\sigma_{1}, d_{1}\right) \ldots\left(\sigma_{n}, d_{n}\right)$ over $\Sigma$ and for all sets $L \subseteq(\Sigma \times \mathbf{R}), \mathcal{T}$ after $\alpha$ MUST $L \Longleftrightarrow$ $\mathcal{T}^{\prime}$ after $\alpha$ MUST $L$.

Example 2. First, contemplate the timed transition systems $\mathcal{T}^{\prime}$ and $\mathcal{T}^{\prime \prime}$ shown in Fig. 2(b) which are not test-equivalent because $\mathcal{T}^{\prime \prime}$ after $(a, 3)(b, 5)$ MUST $\{(a, 6)\}$ but it is not the case for $\mathcal{T}^{\prime}$.

Second, consider the timed transition systems $\widehat{\mathcal{T}}$ and $\check{\mathcal{T}}$ shown in Fig. 2( (c). Notice, they have the same language $\left\{\alpha \mid \alpha \beta=\left(a, d_{1}\right)\left(b, d_{2}\right)\left(c, d_{3}\right), d_{1} \leq 1,1<d_{2}-d_{1}<4,0 \leq d_{3}-d_{2} \leq 3\right\}$. It is easy to see that in both the systems there is a unique run of the empty timed word which can be extended only by an occurrence of an action a at time $d$, where $d \leq 1$. Then, we have that $\widehat{\mathcal{T}}$ after $\epsilon$ MUST $L \Longleftrightarrow \check{\mathcal{T}}$ after $\epsilon$ MUST $L$, only for all sets $L \subseteq(\Sigma \times \mathbf{R})$ containing a pair $(a, d)$, where $d \leq 1$. Moreover, in both the systems for any non-empty timed word from their languages there is the run of the timed word, which cannot be extended. This implies that $\neg(\widehat{\mathcal{T}}$ after $\alpha$ MUST $L) \Longleftrightarrow \neg(\check{\mathcal{T}}$ after $\alpha$ MUST $L)$, for all timed words $\epsilon \neq \alpha \in \mathcal{L}(\widehat{\mathcal{T}})$ and for all sets $L \subseteq(\Sigma \times \mathbf{R})$. Thus, $\widehat{\mathcal{T}}$ and $\check{\mathcal{T}}$ are test-equivalent.

Timed Bisimulation. One of the main advantages of Park-Milner's notion of bisimulation for untimed transition systems, is the fact that the property of being bisimilar may be expressed in terms of presenting an explicit bisimulation between two systems, i.e. a relation on the states of the systems. A timed extension of bisimulation has been extensively studied for timed models (see [26, 28] among others).

Definition 5. Timed transition systems $\mathcal{T}$ and $\mathcal{T}^{\prime}$ over $\Sigma$ are called timed bisimilar equivalent (or bis-equivalent) iff there is a relation $\mathcal{B} \subseteq \operatorname{Conf}(\mathcal{T}) \times \operatorname{Conf}(\mathcal{T})$ such that $\left(\left\langle s_{0}, \nu_{0}\right\rangle,\left\langle s_{0}^{\prime}, \nu_{0}^{\prime}\right\rangle\right) \in \mathcal{B}$ and for all $\left(\langle s, \nu\rangle,\left\langle s^{\prime}, \nu^{\prime}\right\rangle\right) \in \mathcal{B}$ the following holds:

- whenever $\langle s, \nu\rangle \stackrel{\sigma}{\underset{d}{d}}\left\langle s_{1}, \nu_{1}\right\rangle$ in $\mathcal{T}$, then $\left\langle s^{\prime}, \nu^{\prime}\right\rangle \underset{d}{\stackrel{\sigma}{d}}\left\langle s_{1}^{\prime}, \nu_{1}^{\prime}\right\rangle$ in $\mathcal{T}^{\prime}$ and $\left(\left\langle s_{1}, \nu_{1}\right\rangle,\left\langle s_{1}^{\prime}, \nu_{1}^{\prime}\right\rangle\right) \in \mathcal{B}$, for some $\left\langle s_{1}^{\prime}, \nu_{1}^{\prime}\right\rangle$,

- whenever $\left\langle s^{\prime}, \nu^{\prime}\right\rangle \stackrel{\sigma}{\vec{d}}\left\langle s_{1}^{\prime}, \nu_{1}^{\prime}\right\rangle$ in $\mathcal{T}^{\prime}$, then $\langle s, \nu\rangle \stackrel{\sigma}{\underset{d}{\longrightarrow}}\left\langle s_{1}, \nu_{1}\right\rangle$ in $\mathcal{T}$ and $\left(\left\langle s_{1}, \nu_{1}\right\rangle,\left\langle s_{1}^{\prime}, \nu_{1}^{\prime}\right\rangle\right) \in \mathcal{B}$, for some $\left\langle s_{1}, \nu_{1}\right\rangle$. 
Example 3. To illustrate the concept, consider the timed transition systems shown in Fig. 2(c),(d). The systems $\widehat{\mathcal{T}}$ and $\check{\mathcal{T}}$ are not bis-equivalent because, for example, in $\check{\mathcal{T}}$ there exists a run of $(a, 0)$ which can be extended by occurrences of both actions $b$ and $c$ at time 1.2 but it is not the case in $\widehat{\mathcal{T}}$. On the other hand, the timed transition systems $\widetilde{\mathcal{T}}$ and $\overline{\mathcal{T}}$ are bisequivalent because their configurations reachable by runs of same timed words can be extended by occurrences of same actions at same times. For example, in both the systems, the configurations reachable by the runs of the timed word $(a, 2)(b, 3)$ can be extended by an occurrence of an action $c$ at time 5 .

Timed Barbed Bisimulation. Barbed bisimilarity [17 is a widely used concurrency semantics for process algebras that include the silent step ('invisible' action) $\tau$. Barbed bisimulation differs from (strong) bisimulation in the following: 'visible' and 'invisible' actions are distinguished; transitions labelled by 'invisible' actions are required to be bisimulated; only the existence of a transition labelled by a 'visible' action has to be matched. An important feature of barbed bisimulation is that it can be successfully employed when the operational semantics of a process algebra is defined by a reduction relation (i.e., no labels over transitions). It allows one to recover from such a formulation the well-known bisimulation-based equivalences which are defined on labelled transition systems. Another advantage of barbed bisimulation semantics is that it can be described uniformally in different processes calculi (e.g., CCS, $\pi$-calculus, higher order $\pi$-calculus). Recently, the paper [7] has treated timed barbed bisimulation in the context of a timed extension of the $\pi$-calculus.

Introduce auxiliary notions and notations. Given a timed transition system $\mathcal{T}=$ $\left(S, s_{0}, \Sigma_{\tau}, V, T\right)$, a state $s \in S$ is called $\tau$-accessible iff $s_{0} \underset{\delta_{1}, \lambda_{1}}{\stackrel{\tau}{\rightarrow}} s_{1} \ldots s_{n-1} \underset{\delta_{n}, \lambda_{n}}{\stackrel{\tau}{\rightarrow}} s_{n}=s(n \geq 0)$. Define the set $S_{\tau}(\mathcal{T})=\{s \in S \mid s$ is a $\tau$-accessible state $\}$. A configuration $\langle s, \nu\rangle$ of $\mathcal{T}$ is called $\tau$-reachable if there is a run $\left\langle s_{0}, \nu_{0}\right\rangle \underset{d_{1}}{\vec{\tau}}\left\langle s_{1}, \nu_{1}\right\rangle \ldots\left\langle s_{n-1}, \nu_{n-1}\right\rangle \underset{d_{n}}{\stackrel{\tau}{\rightarrow}}\left\langle s_{n}, \nu_{n}\right\rangle=\langle s, \nu\rangle$ of the timed word $\left(\tau, d_{1}\right) \ldots\left(\tau, d_{n}\right)$. We shall use $\operatorname{Conf}_{\tau}(\mathcal{T})$ to denote the set of $\tau$-reachable configurations. From now on, for a configuration $\langle s, \nu\rangle \in \operatorname{Conf}_{\tau}(\mathcal{T})$, we shall write $\langle s, \nu\rangle \underset{d}{\vec{d}}$ iff there is a configuration $\left\langle s^{\prime}, \nu^{\prime}\right\rangle \in \operatorname{Conf}(\mathcal{T})$ such that $\langle s, \nu\rangle \underset{d}{\frac{\sigma}{d}}\left\langle s^{\prime}, \nu^{\prime}\right\rangle$, for some $\sigma \in \Sigma$ and $d \in \mathbf{R}$.

Definition 6. Timed transition systems $\mathcal{T}$ and $\mathcal{T}^{\prime}$ over $\Sigma_{\tau}$ are timed barbed bisimilar equivalent (or bbis-equivalent) iff there is a relation $\mathcal{B} \subseteq \operatorname{Conf}_{\tau}(\mathcal{T}) \times \operatorname{Conf}_{\tau}\left(\mathcal{T}^{\prime}\right)$ such that $\left(\left\langle s_{0}, \nu_{0}\right\rangle,\left\langle s_{0}^{\prime}, \nu_{0}^{\prime}\right\rangle\right) \in \mathcal{B}$ and for all $\left(\langle s, \nu\rangle,\left\langle s^{\prime}, \nu^{\prime}\right\rangle\right) \in \mathcal{B}$ the following holds:

- $\quad-$ if $\langle s, \nu\rangle \underset{d}{\stackrel{\tau}{d}}\left\langle s_{1}, \nu_{1}\right\rangle$ in $\mathcal{T}$, then $\left\langle s^{\prime}, \nu^{\prime}\right\rangle \underset{d}{\stackrel{\tau}{d}}\left\langle s_{1}^{\prime}, \nu_{1}^{\prime}\right\rangle$ in $\mathcal{T}^{\prime}$ and $\left(\left\langle s_{1}, \nu_{1}\right\rangle,\left\langle s_{1}^{\prime}, \nu_{1}^{\prime}\right\rangle\right) \in \mathcal{B}$, for some $\left\langle s_{1}^{\prime}, \nu_{1}^{\prime}\right\rangle$,

- if $\left\langle s^{\prime}, \nu^{\prime}\right\rangle \underset{d}{\stackrel{\tau}{d}}\left\langle s_{1}^{\prime}, \nu_{1}^{\prime}\right\rangle$ in $\mathcal{T}^{\prime}$, then $\langle s, \nu\rangle \underset{d}{\stackrel{\tau}{d}}\left\langle s_{1}, \nu_{1}\right\rangle$ in $\mathcal{T}$ and $\left(\left\langle s_{1}, \nu_{1}\right\rangle,\left\langle s_{1}^{\prime}, \nu_{1}^{\prime}\right\rangle\right) \in \mathcal{B}$, for some $\left\langle s_{1}, \nu_{1}\right\rangle$,

- $\quad$ if $\langle s, \nu\rangle \underset{d}{\stackrel{\sigma}{\longrightarrow}}(\sigma \in \Sigma)$ in $\mathcal{T}$, then $\left\langle s^{\prime}, \nu^{\prime}\right\rangle \underset{d}{\stackrel{\sigma^{\prime}}{\longrightarrow}}\left(\sigma^{\prime} \in \Sigma\right)$ in $\mathcal{T}^{\prime}$,

- if $\left\langle s^{\prime}, \nu^{\prime}\right\rangle \underset{d}{\stackrel{\sigma}{\longrightarrow}}(\sigma \in \Sigma)$ in $\mathcal{T}^{\prime}$, then $\langle s, \nu\rangle \underset{d}{\stackrel{\sigma^{\prime}}{\longrightarrow}}\left(\sigma^{\prime} \in \Sigma\right)$ in $\mathcal{T}$.

Example 4. The timed transition system $\mathcal{T}$ depicted in Fig. 1 and the timed transition system $\dot{\mathcal{T}}$ depicted at the left side of Fig. 3 are not bbis-equivalent because, for example, in $\dot{\mathcal{T}}$ there exists the run of the empty timed word which can be extended by an occurrence of an action $\tau$ at time 0 but it is not the case in $\mathcal{T}$. On the other hand, Fig. 3 shows the bbis-equivalent 

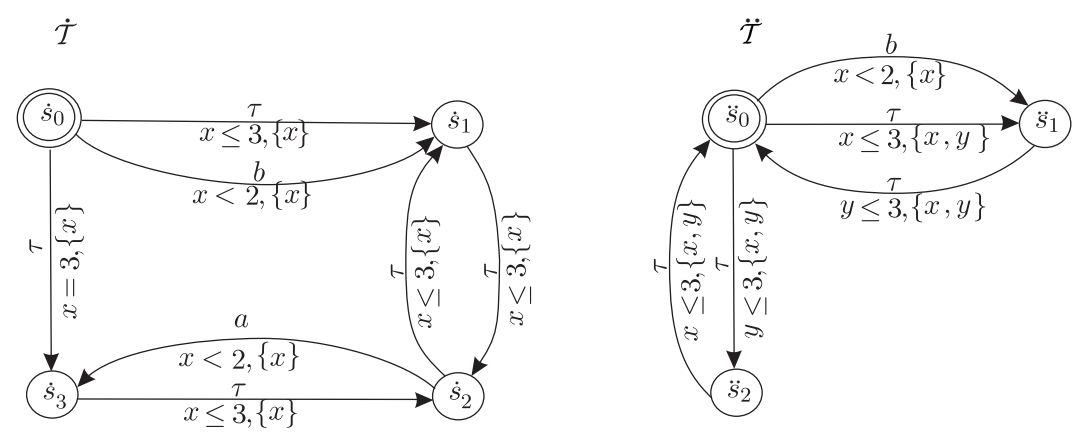

Figure 3: An example of bbis-equivalent timed transition systems

timed transition systems $\dot{\mathcal{T}}$ and $\ddot{\mathcal{T}}$. The configurations of these systems, reachable by the runs of $\left(\tau, d_{1}\right) \ldots\left(\tau, d_{n}\right)(n \geq 0)$, can be extended by an occurrence of an action $\tau$ at same time, and by an occurrence of a non- $\tau$-action at same time, if $n=2 i(i \geq 0)$. For example, in both the timed transition systems, the configurations reachable by the runs of $(\tau, 1)(\tau, 2)$ can be extended by an occurrence of an action $\tau$ at time 4 and by an occurrence of some $\sigma \in\{a, b\}$ at time 3 .

\section{Open Maps Bisimulation}

\subsection{Preliminaries}

The concept of open map (open morphism) appears in work of Joyal and Moerdijk 13 where a notion of a subcategory of open maps of a (pre)topos is defined. As reported in [14, the open map approach provides general concepts of bisimilarity for any categorical model of computations.

First, a category $\mathbb{M}$ whose objects represent models has to be identified. A morphism $m: X \longrightarrow Y$ in $\mathbb{M}$ should intuitively be thought of as a simulation of the object $X$ in the object $Y$. Then, inside the category $\mathbb{M}$, a subcategory $\mathbb{P}$ of 'path objects' and 'path extension' morphisms between these objects is to be chosen. Given a path object $P$ in $\mathbb{P}$ and a model object $X$ in $\mathbb{M}$, a path is a morphism $p: P \longrightarrow X$ in $\mathbb{M}$. We think of $p$ as representing a particular way of realizing $P$ in $X$.

Second, we have to identify morphisms $m: X \longrightarrow Y$ which have the property that whenever a computation of $X$ can be extended via $m$ in $Y$ then that extension can be matched by an extension of the computation in $X$. A morphism $m: X \rightarrow Y$ in $\mathbb{M}$ is called $\mathbb{P}$-open iff whenever $f: P_{1} \rightarrow P_{2}$ in $\mathbb{P}, p: P_{1} \rightarrow X$ and $q: P_{2} \rightarrow Y$ in $\mathbb{M}$, and $m \circ p=q \circ f$, then there exists a morphism $h: P_{2} \rightarrow X$ in $\mathbb{M}$ such that $p=h \circ f$ and $q=m \circ h$.

Third, an abstract notion of bisimilarity has to be introduced. The definition is given in terms of spans of open maps. Two objects $X$ and $Y$ in $\mathbb{M}$ are said to be $\mathbb{P}$-bisimilar if there exists a span $X \stackrel{m}{\longleftarrow} Z \stackrel{m^{\prime}}{\longrightarrow} Y$ with a common object $Z$ of $\mathbb{P}$-open morphisms.

In the following we will reformulate the behavioral equivalences on timed transition systems by varying categories and subcategories of the model. 


\subsection{Open Maps Characterizations}

In this subsection, different categories of timed transition systems are introduced and open maps based characterizations of the behavioral equivalences are given.

We start with introducing some auxiliary notations. For a timed transition system $\mathcal{T}$ over $\Sigma$, define the following:

- for $\gamma=\left\langle s_{0}, \nu_{0}\right\rangle \underset{d_{1}}{\sigma_{1}} \ldots \frac{\sigma_{n}}{d_{n}}\left\langle s_{n}, \nu_{n}\right\rangle \in \operatorname{Runs}(\mathcal{T}), t w(\gamma)=\left(\sigma_{1}, d_{1}\right) \ldots\left(\sigma_{n}, d_{n}\right)$ and $\mathcal{A}_{\mathcal{T}}(\gamma)=$ $\left\{\left(\sigma_{n+1}, d_{n+1}\right) \mid \exists\left\langle s_{n+1}, \nu_{n+1}\right\rangle\right.$ s.t. $\left.\left\langle s_{n}, \nu_{n}\right\rangle \underset{d_{n+1}}{\stackrel{\sigma_{n+1}}{\longrightarrow}}\left\langle s_{n+1}, \nu_{n+1}\right\rangle\right\}$,

- $\mathcal{S R}(\mathcal{T})$ is the least subset of $\left(2^{\operatorname{Runs}(\mathcal{T})} \backslash\{\emptyset\}\right)$ such that $\forall \gamma \in \operatorname{Runs}(\mathcal{T}) \exists X \in \mathcal{S R}(\mathcal{T})$ 。 $\gamma \in X$, and $\forall X \in \mathcal{S R}(\mathcal{T}) \forall \gamma, \gamma^{\prime} \in X \diamond t w(\gamma)=t w\left(\gamma^{\prime}\right)$,

- for $X \in \mathcal{S R}(\mathcal{T}), \mathcal{A}_{\mathcal{T}}(X)=\left\{\mathcal{A}_{\mathcal{T}}(\gamma) \mid \gamma \in X\right\}$, tw $(X)=t w(\gamma)$ for some $\gamma \in X$, and $\mathcal{A}_{\mathcal{T}}(\alpha)=\mathcal{A}_{\mathcal{T}}(X)$, if $t w(X)=\alpha$.

We are ready to define a number of categories of timed transition systems.

Category $\mathbf{T} \mathbf{T} \mathbf{S}_{\text {trace }}$. The objects of this category are the timed transition systems over $\Sigma$ (see Definition 1). Guided by our intuitive understanding how the timed words generated by the runs of a system can be simulated, we define the morphisms between timed transition systems as follows.

Definition 7. Given timed transition systems $\mathcal{T}$ and $\mathcal{T}^{\prime}$ over $\Sigma$, the $t_{\text {trace-morphism between }} \mathcal{T}$ and $\mathcal{T}^{\prime}$ is a mapping $\mu: \mathcal{S R}(\mathcal{T}) \rightarrow \mathcal{S R}\left(\mathcal{T}^{\prime}\right)$ such that $t w(X)=t w(\mu(X))$, for all $X \in \mathcal{S} \mathcal{R}(\mathcal{T})$.

Example 5. Consider $\mathcal{T}^{\star}$ and $\mathcal{T}^{*}$ depicted in Fig. 2(a). From Example 1, we know that $\mathcal{L}\left(\mathcal{T}^{\star}\right) \subset \mathcal{L}\left(\mathcal{T}^{*}\right)$. Then, for each set $X^{\star} \in \mathcal{S R}\left(\mathcal{T}^{\star}\right)$ with $t w\left(X^{\star}\right)=\alpha \in \mathcal{L}\left(\mathcal{T}^{\star}\right)$, we can find the set $X^{*} \in \mathcal{S R}\left(\mathcal{T}^{*}\right)$ with $t w\left(X^{*}\right)=\alpha \in \mathcal{L}\left(\mathcal{T}^{*}\right)$. For example, the set $X^{\star} \in \mathcal{S} \mathcal{R}\left(\mathcal{T}^{\star}\right)$ with $t w\left(X^{\star}\right)=(a, 1)(b, 1)(a, 1)$ can be connected to the set $X^{*} \in \mathcal{S R}\left(\mathcal{T}^{*}\right)$ with $\operatorname{tw}\left(X^{*}\right)=$ $(a, 1)(b, 1)(a, 1)$. Hence, we can easily specify a trace-morphism, say, $\mu^{\star}$ from $\mathcal{T}^{\star}$ to $\mathcal{T}^{*}$.

Timed transition systems over an alphabet $\Sigma$ and $t_{\text {trace-morphisms between them form }}$ a category $\mathbf{T} \mathbf{T} \mathbf{S}_{\text {trace }}$ in which the composition of two $t_{\text {trace }}$-morphisms $\mu_{1}: \mathcal{T}_{0} \longrightarrow \mathcal{T}_{1}$ and $\mu_{2}: \mathcal{T}_{1} \longrightarrow \mathcal{T}_{2}$ is defined as $\left(\mu_{2} \circ \mu_{1}\right): \mathcal{T}_{0} \longrightarrow \mathcal{T}_{2}$, and the identity $t_{\text {trace }}$-morphism is the identity function.

Next step is to choose a subcategory $\mathbf{P}_{\text {trace }}$ with path objects - timed transition systems corresponding to timed words, and with paths - morphisms of the category $\mathbf{T} \mathbf{T} \mathbf{S}_{\text {trace }}$.

Definition 8. The full subcategory $\mathbf{P}_{\text {trace }}$ of the category $\mathbf{T} \mathbf{T} \mathbf{S}_{\text {trace }}$ contains objects $\mathcal{T}^{\alpha}=$ $\left(S^{\alpha}, 0, \Sigma, V^{\alpha}, T^{\alpha}\right)$ corresponding to timed words $\alpha=\left(\sigma_{1}, d_{1}\right) \ldots\left(\sigma_{n}, d_{n}\right)(n \geq 0)$ over $\Sigma$, where $S^{\alpha}=\{0,1 . .,(n-1), n\}$ with the initial state $0, V^{\alpha}=\{u\}, T^{\alpha}=\left\{(i-1) \underset{u=d_{i}, \emptyset}{\stackrel{\sigma_{i}}{\rightarrow}} i \mid i=1 \ldots n\right\}$,

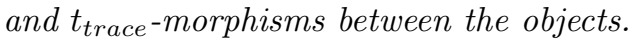

The following auxiliary facts will be helpful to establish some results.

Lemma 1. Given an object $\mathcal{T}^{\alpha}$ in $\mathbf{P}_{\text {trace }}$ and an object $\mathcal{T}$ in $\mathbf{T} \mathbf{T} \mathbf{S}_{\text {trace }}$,

(i) there exists $X \in \mathcal{S R}\left(\mathcal{T}^{\alpha}\right)$ with $\operatorname{tw}(X)=\alpha$,

(ii) there is a bijection between the timed words $\beta \in \mathcal{L}(\mathcal{T})$ and the sets $X \in \mathcal{S R}(\mathcal{T})$ with $t w(X)=\beta$, 


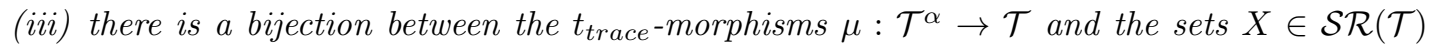
with $\operatorname{tw}(X)=\alpha$.

Consider a behavioral characterization of the notion of open maps corresponding to the subcategory $\mathbf{P}_{\text {trace }}$.

Proposition 1. Let $\mathcal{T}, \mathcal{T}^{\prime}$ be objects in $\mathbf{T} \mathbf{T} \mathbf{S}_{\text {trace }}$. Then, a morphism $\mu: \mathcal{T} \rightarrow \mathcal{T}^{\prime}$ in $\mathbf{T T S}_{\text {trace }}$

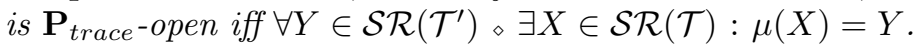

Example 6. As shown in Example 5, there is a trace-morphism $\mu^{\star}$ from $\mathcal{T}^{\star}$ to $\mathcal{T}^{*}$ depicted in Fig. 2(a). However, for the set $X^{*} \in \mathcal{S R}\left(\mathcal{T}^{*}\right)$ with $t w\left(X^{*}\right)=(a, 3)(b, 4)(a, 5)$, we cannot find any set $X^{\star} \in \mathcal{S R}\left(\mathcal{T}^{\star}\right)$ with $t w\left(X^{\star}\right)=(a, 3)(b, 4)(a, 5)$. Hence, $\mu^{\star}$ is not a $\mathbf{P}_{\text {trace-open }}$ morphism, by Proposition 1. From example 1, we know that the languages of the systems $\mathcal{T}^{\prime}$ and $\mathcal{T}^{\prime \prime}$ shown in Fig. 2(b) coincide. Then, due to Lemma $1\left(\right.$ ii), for any set $X^{\prime} \in \mathcal{S R}\left(\mathcal{T}^{\prime}\right)$ with $t w\left(X^{\prime}\right)=\alpha \in \mathcal{L}\left(\mathcal{T}^{\prime}\right)$, there is a set $X^{\prime \prime} \in \mathcal{S R}\left(\mathcal{T}^{\prime \prime}\right)$ with $t w\left(X^{\prime \prime}\right)=\alpha \in \mathcal{L}\left(\mathcal{T}^{\prime \prime}\right)$, and vice versa. For example, for the set $X^{\prime}$ with $t w\left(X^{\prime}\right)=(a, 1)(b, 3)$ we can find the set $X^{\prime \prime} \in \mathcal{S} \mathcal{R}\left(\mathcal{T}^{\prime \prime}\right)$ such that $t w\left(X^{\prime \prime}\right)=(a, 1)(b, 3)$ and for the set $Y^{\prime \prime} \in \mathcal{S R}\left(\mathcal{T}^{\prime \prime}\right)$ with $t w(Y)=(a, 2)$ we have the set

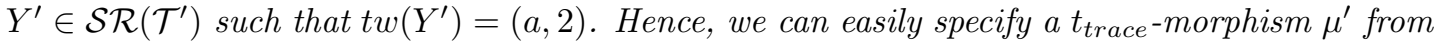

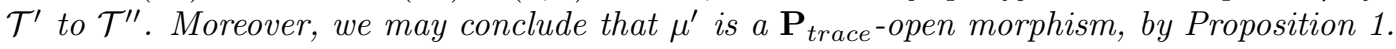

Category $\mathbf{T T S}_{\text {test }}$. We modify the category $\mathbf{T} \mathbf{T S} \mathbf{S}_{\text {trace }}$ only with respect to the morphisms. The morphisms of the category $\mathbf{T} \mathbf{T} \mathbf{S}_{t e s t}$ reflect not only correspondences between the timed words of systems but also matches of the sets of actions with times at which the actions occur after executions of the timed words.

Definition 9. Given timed transition systems $\mathcal{T}$ and $\mathcal{T}^{\prime}$ over $\Sigma$, the $t_{\text {test }}$-morphism between $\mathcal{T}$ and $\mathcal{T}^{\prime}$ is a mapping $\mu: \mathcal{S R}(\mathcal{T}) \rightarrow \mathcal{S R}\left(\mathcal{T}^{\prime}\right)$ such that for all $X \in \mathcal{S R}(\mathcal{T})$ the following holds $t w(X)=t w(\mu(X))$ and $\forall A^{\prime} \in \mathcal{A}_{\mathcal{T}^{\prime}}(\mu(X)) \exists A \in \mathcal{A}_{\mathcal{T}}(X) \diamond A \subseteq A^{\prime}$.

Example 7. Investigate $\mathcal{T}^{\prime}$ and $\mathcal{T}^{\prime \prime}$ depicted in Fig. 2(b). From examples 1 and 6, we know that the languages of the systems coincide and there is a trace-morphism $\mu^{\prime}$ from $\mathcal{T}^{\prime}$ to $\mathcal{T}^{\prime \prime}$. The latter means that for any set $X^{\prime} \in \mathcal{S R}\left(\mathcal{T}^{\prime}\right)$ it holds that $t w\left(X^{\prime}\right)=t w\left(\mu^{\prime}\left(X^{\prime}\right)\right)$. Take an arbitrary $\alpha \in \mathcal{L}\left(\mathcal{T}^{\prime \prime}\right)$. It is easy to see that for all runs $\gamma^{\prime \prime}$ of $\alpha$ in $\mathcal{T}^{\prime \prime}$, there is a run $\gamma^{\prime}$ of $\alpha$ in $\mathcal{T}^{\prime}$ such that $\mathcal{A}_{\mathcal{T}^{\prime}}\left(\gamma^{\prime}\right)=\mathcal{A}_{\mathcal{T}^{\prime \prime}}\left(\gamma^{\prime \prime}\right)$. For example, for the run $\gamma^{\prime \prime}=\left\langle s_{0}^{\prime \prime}, \nu_{0}^{\prime \prime}\right\rangle \underset{2}{\vec{a}}\left\langle s_{1}^{\prime \prime}, \nu_{1}^{\prime \prime}\right\rangle$ $\underset{2}{\stackrel{b}{\longrightarrow}}\left\langle s_{2}^{\prime \prime}, \nu_{0}^{\prime \prime}\right\rangle$ in $\mathcal{T}^{\prime \prime}$ with $\mathcal{A}_{\mathcal{T}^{\prime \prime}}\left(\gamma^{\prime \prime}\right)=\{(a, d) \mid 2 \leq d \leq 5\}$ we can find the run $\gamma^{\prime}=\left\langle s_{0}^{\prime}, \nu_{0}^{\prime}\right\rangle \stackrel{a}{\overrightarrow{2}}$ $\left\langle s_{1}^{\prime}, \nu_{1}^{\prime}\right\rangle \stackrel{b}{\rightarrow}\left\langle s_{0}^{\prime}, \nu_{0}^{\prime}\right\rangle$ in $\mathcal{T}^{\prime}$ such that $\mathcal{A}_{\mathcal{T}^{\prime}}\left(\gamma^{\prime}\right)=\{(a, d) \mid 2 \leq d \leq 5\}$. Then, we can say that for all $A^{\prime \prime} \in \mathcal{A}_{\mathcal{T}^{\prime \prime}}\left(\mu\left(X^{\prime}\right)\right)$ there exists $A^{\prime} \in \mathcal{A}_{\mathcal{T}}{ }^{\prime}\left(X^{\prime}\right)$ such that $A^{\prime}=A^{\prime \prime}$. Hence, one can easily specify a $t_{\text {test }}$-morphism $\mu^{\prime \prime}$ from $\mathcal{T}^{\prime}$ to $\mathcal{T}^{\prime \prime}$.

Timed transition systems over an alphabet $\Sigma$ and $t_{\text {test }}$-morphisms between them form a category $\mathbf{T T S}_{\text {test }}$, in which the composition of two $t_{\text {test }}$-morphisms $\mu: \mathcal{T} \rightarrow \mathcal{T}^{\prime}$ and $\mu^{\prime}: \mathcal{T}^{\prime} \rightarrow$ $\mathcal{T}^{\prime \prime}$ is $\mu^{\prime} \circ \mu: \mathcal{T} \rightarrow \mathcal{T}^{\prime \prime}$, and the identity $t_{\text {test }}$-morphism is the identity function.

Next, we define a subcategory of path objects which are trees consisting of a trunk and branches of length one, corresponding to a timed word, and a more general branching structure, corresponding a set of actions with times at which the actions occur after an execution of the timed word.

Definition 10. The full subcategory $\mathbf{P}_{\text {test }}$ of the category $\mathbf{T} \mathbf{T S}_{\text {test }}$ contains objects $\mathcal{T}^{\alpha, L}$ corresponding to a timed word $\alpha=\left(\sigma_{1}, d_{1}\right) \ldots\left(\sigma_{n}, d_{n}\right)$ over $\Sigma$ and a set $L=\left\{\left(a_{1}, d_{1}^{\prime}\right), \ldots,\left(a_{k}, d_{k}^{\prime}\right)\right.$, $\ldots\} \subseteq(\Sigma \times \mathbf{R})$ of the form 


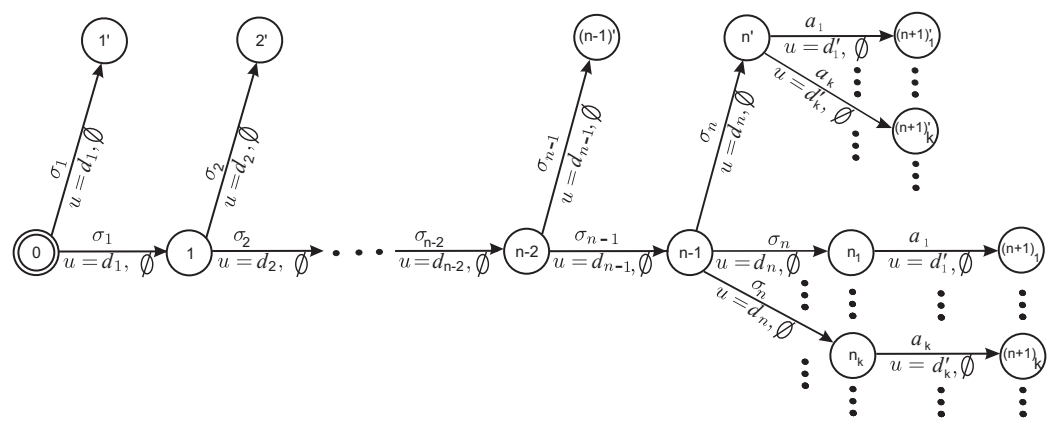

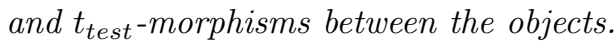

The following facts will allow us to provide a behavioral characterization of open maps corresponding the subcategory $\mathbf{P}_{\text {test }}$.

Lemma 2. Given an object $\mathcal{T}^{\alpha, L}$ in $\mathbf{P}_{\text {test }}$ and an object $\mathcal{T}$ in $\mathbf{T} \mathbf{T} \mathbf{S}_{\text {test }}$,

(i) there exists $X \in \mathcal{S R}\left(\mathcal{T}^{\alpha, L}\right)$ with $t w(X)=\alpha$ and $Y \in \mathcal{S R}\left(\mathcal{T}^{\alpha, L}\right)$ with $t w(Y)=\alpha(a, d)$, for all $(a, d) \in L$,

(ii) there exists a bijection between the timed words $\beta \in \mathcal{L}(\mathcal{T})$ and the sets $X \in \mathcal{S} \mathcal{R}(\mathcal{T})$ with $t w(X)=\beta$,

(iii) there exists a bijection between the $t_{\text {test }}$-morphisms $\mu: \mathcal{T}^{\alpha, L} \rightarrow \mathcal{T}$ and the pairs $(Y, L)$ such that $Y \in \mathcal{S R}(\mathcal{T}), t w(Y)=\alpha$, and $L \subseteq \bigcup_{A \in \mathcal{A}_{\mathcal{T}}(Y)} A$, for all $A \in \mathcal{A}_{\mathcal{T}}(Y) A \cap L \neq \emptyset$, if $L \neq \emptyset$.

Our next aim is to characterize $\mathbf{P}_{\text {test }}$-open morphisms.

Proposition 2. Let $\mathcal{T}, \mathcal{T}^{\prime}$ be objects in $\mathbf{T T S}_{\text {test }}$. Then, a morphism $\mu: \mathcal{T} \rightarrow \mathcal{T}^{\prime}$ in $\mathbf{T T S}_{\text {test }}$

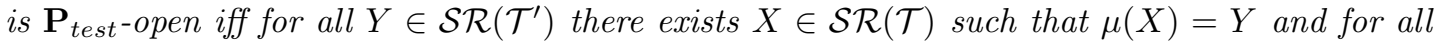
$A \in \mathcal{A}_{\mathcal{T}}(X)$ there exists $A^{\prime} \in \mathcal{A}_{\mathcal{T}^{\prime}}(Y)$ such that $A^{\prime} \subseteq A$.

Example 8. As shown in Examples 20 and 7 , the timed transition systems $\mathcal{T}^{\prime}$ and $\mathcal{T}^{\prime \prime}$ depicted in Fig. 2(b) have the same languages and there is a test-morphism $\mu^{\prime \prime}$ from $\mathcal{T}^{\prime}$ to $\mathcal{T}^{\prime \prime}$. Contemplate the run $\gamma^{\prime}=\left\langle s_{0}^{\prime}, \nu_{0}^{\prime}\right\rangle \stackrel{a}{\rightarrow}\left\langle s_{1}^{\prime}, \nu_{0}^{\prime}\right\rangle \stackrel{b}{\rightarrow}\left\langle s_{2}^{\prime}, \nu_{0}^{\prime}\right\rangle$ with $\mathcal{A}_{\mathcal{T}^{\prime}}\left(\gamma^{\prime}\right)=\emptyset$ in $\mathcal{T}^{\prime}$. It is easy to see that in $\mathcal{T}^{\prime \prime}$ there is no run $\gamma^{\prime \prime}$ of $(a, 0)(b, 2)$ with $\mathcal{A}_{\mathcal{T}^{\prime \prime}}\left(\gamma^{\prime \prime}\right)=\emptyset$. The above saying implies that for $X^{\prime \prime} \in \mathcal{S R}\left(\mathcal{T}^{\prime \prime}\right)$ with $t w\left(X^{\prime \prime}\right)=(a, 0)(b, 2)$ we can find $X^{\prime} \in \mathcal{S R}\left(\mathcal{T}^{\prime}\right)$ with $t w\left(X^{\prime}\right)=(a, 0)(b, 2)$, however, there is $\emptyset=A^{\prime} \in \mathcal{A}_{\mathcal{T}^{\prime}}\left(X^{\prime}\right)$ such that $\neg\left(A^{\prime \prime} \subseteq A^{\prime}\right)$ for all $A^{\prime \prime} \in \mathcal{A}_{\mathcal{T}^{\prime \prime}}\left(X^{\prime \prime}\right)$. Using Proposition 2, we may conclude that $\mu^{\prime \prime}$ is not a $\mathbf{P}_{\text {test-open morphism. Next, treat the systems }}$ $\widehat{\mathcal{T}}$ and $\check{\mathcal{T}}$ drawn in Fig. 2(c). From Example 2, we know that they have the same languages. Then, by Lemma 2(ii), for any set $\widehat{X} \in \mathcal{S R}(\widehat{\mathcal{T}})$ with $t w(\widehat{X})=\alpha \in \mathcal{L}(\widehat{\mathcal{T}})$, there is a set $\check{X} \in \mathcal{S R}(\check{\mathcal{T}})$ with $t w(\check{X})=\alpha \in \mathcal{L}(\check{\mathcal{T}})$, and vice versa. For example, for the set $\widehat{X} \in \mathcal{S R}(\widehat{\mathcal{T}})$ with $t w(\widehat{X})=(a, 1)$ we can find the set $\check{X} \in \mathcal{S R}(\check{\mathcal{T}})$ consisting of three runs of $(a, 1)$, and for the set $\check{Y} \in \mathcal{S R}(\check{\mathcal{T}})$ with $(a, 0)(b, 2)$ there is the set $\widehat{Y} \in \mathcal{S R}(\widehat{\mathcal{T}})$ including exactly two runs of $(a, 0)(b, 2)$. Also, it is easy to see that $\mathcal{A}_{\tilde{\mathcal{T}}}\left(\left\langle\check{s_{0}}, \check{\nu_{0}}\right\rangle\right)=\mathcal{A}_{\widehat{\mathcal{T}}}\left(\left\langle\widehat{s_{0}}, \widehat{\nu_{0}}\right\rangle\right)$. Moreover, for all non-empty timed words $\alpha$ in $\mathcal{L}(\widehat{\mathcal{T}})$, in $\check{\mathcal{T}}$ we can find a run $\check{r}$ of $\alpha$ such that $\mathcal{A}_{\check{\mathcal{T}}}(\check{r})=\emptyset$, and vice versa. For example, for the timed word $(a, 0) \in \mathcal{L}(\widehat{\mathcal{T}})$, in $\check{\mathcal{T}}$ there is the run $\check{\gamma}=\left\langle\check{s_{0}}, \check{\nu_{0}}\right\rangle \stackrel{a}{\rightarrow}\left\langle\check{s_{3}}, \check{\nu_{1}}\right\rangle$ 
such that $\mathcal{A}_{\breve{\mathcal{T}}}(\check{\gamma})=\emptyset$. Further, for the timed word $(a, 1)(b, 3) \in \mathcal{L}(\check{\mathcal{T}})$, in $\widehat{\mathcal{T}}$ we have the run $\widehat{\gamma}=\left\langle\widehat{s_{0}}, \widehat{\nu_{0}}\right\rangle \stackrel{a}{\rightarrow}\left\langle\widehat{s_{1}}, \widehat{\nu_{1}}\right\rangle \stackrel{b}{\rightarrow}\left\langle\widehat{s_{4}}, \widehat{\nu_{0}}\right\rangle$ such that $\mathcal{A}_{\widehat{\mathcal{T}}}(\widehat{\gamma})=\emptyset$. Thus, we can easily specify a $\mathbf{P}_{\text {test }}$-open morphism $\widehat{\mu}$ from $\widehat{\mathcal{T}}$ to $\check{\mathcal{T}}$, due to Proposition 2 .

Category $\mathbf{T T S}_{b i s}$. This category contains objects which are timed transition systems over an alphabet $\Sigma$ and morphisms which represent some notions of simulation of the behavior of one system by the other. This leads to the following definition of a morphism consisting of two functions, one mapping states of the simulated system to simulating states of the other, and one mapping clocks of the simulating system to simulated clocks.

Definition 11. Given timed transition systems $\mathcal{T}=\left(S, s_{0}, \Sigma, V, T\right)$ and $\mathcal{T}^{\prime}=\left(S^{\prime}, s_{0}^{\prime}\right.$, $\left.\Sigma, V^{\prime}, T^{\prime}\right)$ over $\Sigma$, a pair $(\mu, \eta)$ is a $t_{b i s}$-morphism between $\mathcal{T}$ and $\mathcal{T}^{\prime}$, if $\mu: S \rightarrow S^{\prime}$ is a mapping between the states, and $\eta: V^{\prime} \rightarrow V$ is a mapping between the clock variables, satisfying

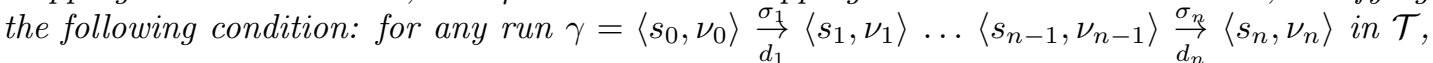
$\left\langle\mu\left(s_{0}\right), \eta^{-1}\left(\nu_{0}\right)\right\rangle \stackrel{\sigma_{1}}{d_{1}}\left\langle\mu\left(s_{1}\right), \eta^{-1}\left(\nu_{1}\right)\right\rangle \ldots\left\langle\mu\left(s_{n-1}\right), \eta^{-1}\left(\nu_{n-1}\right)\right\rangle \underset{d_{n}}{\stackrel{\sigma_{n}}{d_{n}}}\left\langle\mu\left(s_{n}\right), \eta^{-1}\left(\nu_{n}\right)\right\rangle$ is a run in $\mathcal{T}^{\prime}$.

Example 9. Consider the systems $\widetilde{\mathcal{T}}$ and $\overline{\mathcal{T}}$ shown in Fig. 2(d). Construct a mapping $\widetilde{\mu}$ : $S_{\widetilde{\mathcal{T}}} \rightarrow S_{\overline{\mathcal{T}}}$ as follows: $\widetilde{\mu}\left(\widetilde{s_{j}}\right)=\overline{s_{j}}(0 \leq j \leq 4), \widetilde{\mu}\left(\widetilde{s_{5}}\right)=\overline{s_{0}}, \widetilde{\mu}\left(\widetilde{s_{6}}\right)=\overline{s_{2}}$, and take the identical mapping $\widetilde{\eta}: V_{\overline{\mathcal{T}}} \rightarrow V_{\widetilde{\mathcal{T}}}$. Clearly, $\widetilde{\mu}$ and $\widetilde{\eta}$ are indeed mappings. Also, it is easy to see that the $(\widetilde{\mu}, \widetilde{\eta})$-image of each run in $\widetilde{\mathcal{T}}$ is a run in $\overline{\mathcal{T}}$. For example, the $(\widetilde{\mu}, \widetilde{\eta})$-image of the run $\left\langle\widetilde{s_{0}}, \widetilde{\nu_{0}}\right\rangle$ $\underset{1}{\stackrel{a}{\rightarrow}}\left\langle\widetilde{s_{1}}, \widetilde{\nu_{1}}\right\rangle \stackrel{b}{\rightarrow}\left\langle\widetilde{s_{6}}, \widetilde{\nu_{0}}\right\rangle$ in $\widetilde{\mathcal{T}}$ is the run $\left\langle\overline{s_{0}}, \overline{\nu_{0}}\right\rangle \stackrel{a}{\rightarrow}\left\langle\overline{s_{1}}, \overline{\nu_{1}}\right\rangle \underset{2}{\stackrel{b}{\rightarrow}}\left\langle\overline{s_{2}}, \overline{\nu_{0}}\right\rangle$ in $\overline{\mathcal{T}}$. Then, the pair $(\widetilde{\mu}, \widetilde{\eta})$ is a $t_{\text {bis }}$-morphism.

Timed transition systems over an alphabet $\Sigma$ and $t_{b i s}$-morphisms between them form a category of timed transition systems, $\mathbf{T} \mathbf{T S} \mathbf{S}_{b i s}$, in which the composition of two morphisms $(\mu, \eta): \mathcal{T} \rightarrow \mathcal{T}^{\prime}$ and $\left(\mu^{\prime}, \eta^{\prime}\right): \mathcal{T}^{\prime} \rightarrow \mathcal{T}^{\prime \prime}$ is defined as $\left(\mu^{\prime}, \eta^{\prime}\right) \circ(\mu, \eta):=\left(\mu^{\prime} \circ \mu, \eta \circ \eta^{\prime}\right)$, and the identity $t_{b i s}$-morphism is a pair of the identity functions.

We would like to choose timed words over $\Sigma$ with word extensions as path objects with morphisms between them so as to form a subcategory of the category $\mathbf{T} \mathbf{T} \mathbf{S}_{b i s}$.

Definition 12. The full subcategory $\mathbf{P}_{\text {bis }}$ of the category $\mathbf{T} \mathbf{T S} \mathbf{S}_{b i s}$ contains objects $\mathcal{T}^{\alpha}=$ $\left(S^{\alpha}, 0, \Sigma, V^{\alpha}, T^{\alpha}\right)^{2}$ corresponding to timed words $\alpha=\left(\sigma_{1}, d_{1}\right) \ldots\left(\sigma_{n}, d_{n}\right)$ over $\Sigma$, where $S^{\alpha}=$ $\{0,1, \ldots,(n-1), n\}$ with the initial state $0, V^{\alpha}$ consists of the $2^{n}$ subsets of states $\{1,2, \ldots, n\}$, $T^{\alpha}=\left\{(i-1) \underset{\delta_{i}, \lambda_{i}}{\stackrel{\sigma_{i}}{\rightarrow}} i \mid i=1 \ldots n, \lambda_{i}=\left\{x \in V^{\alpha} \mid i \in x\right\}, \delta_{i}=\underset{x \in V^{\alpha}}{\wedge}\left(x=d_{i}-d_{I(i, x)}\right)\right\}$, where $I(i, x):=\max \{k \in x \cup\{0\} \mid k<i\}^{3}$ and $d_{0}:=0$, and $t_{b i s}$-morphisms between the objects.

Consider important properties of the objects and morphisms of the category and subcategory under consideration.

Lemma 3. Given an object $\mathcal{T}^{\alpha}$ in $\mathbf{P}_{\text {bis }}$ and an object $\mathcal{T}$ in $\mathbf{T} \mathbf{T S}$ bis,

(i) there is a unique run of $\alpha$ in $\mathcal{T}^{\alpha}$,

(ii) there is a bijection between the runs $\gamma$ of $\alpha$ in $\mathcal{T}$ and the $t_{\text {bis }}$-morphisms $(\mu, \eta): \mathcal{T}^{\alpha} \rightarrow \mathcal{T}$ such that the runs $\gamma$ are the $(\mu, \eta)$-images of the run of $\alpha$ in $\mathcal{T}^{\alpha}$.

\footnotetext{
${ }^{2}$ The construction of $\mathcal{T}^{\alpha}$ has been developed in [11.

${ }^{3}$ The number returned by $I(i, x)$ is the last state before $i$, at which $x$ was reset.
} 


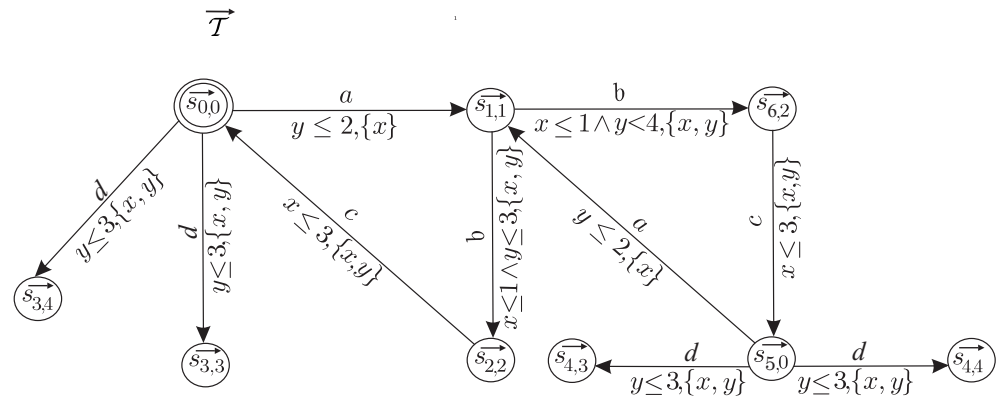

Figure 4: An example of a vertex of span between timed transition systems

Next, we provide a behavioral characterization of $\mathbf{P}_{b i s}$-open morphisms.

Proposition 3. Let $\mathcal{T}, \mathcal{T}^{\prime}$ be objects in $\mathbf{T T S}_{\text {bis }}$. A morphism $(\mu, \eta): \mathcal{T} \longrightarrow \mathcal{T}^{\prime}$ in $\mathbf{T T S}_{\text {bis }}$ is $\mathbf{P}_{\text {bis }}$-open iff for any run $\gamma=\left\langle s_{0}, \nu_{0}\right\rangle \underset{d_{1}}{\stackrel{\sigma_{1}}{\rightarrow}}\left\langle s_{1}, \nu_{1}\right\rangle \ldots\left\langle s_{n-1}, \nu_{n-1}\right\rangle \underset{d_{n}}{\sigma_{n}}\left\langle s_{n}, \nu_{n}\right\rangle$ in $\mathcal{T}$, whenever $\left\langle\mu\left(s_{n}\right), \eta^{-1}\left(\nu_{n}\right)\right\rangle \stackrel{\sigma}{\rightarrow}\left\langle s^{2}, \nu^{2}\right\rangle$ in $\mathcal{T}^{\prime}$, then $\left\langle s_{n}, \nu_{n}\right\rangle \stackrel{\sigma}{\rightarrow}\left\langle s^{1}, \nu^{1}\right\rangle$ in $\mathcal{T}$ and $\left\langle\mu\left(s^{1}\right), \eta^{-1}\left(\nu^{1}\right)\right\rangle=\left\langle s^{2}, \nu^{2}\right\rangle$.

Example 10. Consider the $t_{\text {bis }}$-morphism $(\widetilde{\mu}, \widetilde{\eta}): \widetilde{\mathcal{T}} \rightarrow \overline{\mathcal{T}}$ specified in Example 9 . Clearly, the run $\bar{\gamma}=\left\langle\overline{s_{0}}, \overline{\nu_{0}}\right\rangle$ in $\overline{\mathcal{T}}$ is the $(\widetilde{\mu}, \widetilde{\eta})$-image of the run $\widetilde{\gamma}=\left\langle\widetilde{s_{0}}, \widetilde{\nu_{0}}\right\rangle$ in $\widetilde{\mathcal{T}}$. It is easy to see that $\bar{\gamma}$ can be extended by an occurrence of an action d at time 0 up to the run $\overline{\gamma_{1}}=\left\langle\overline{s_{0}}, \overline{\nu_{0}}\right\rangle \stackrel{d}{\rightarrow}\left\langle\overline{s_{4}}, \overline{\nu_{0}}\right\rangle$, but in $\widetilde{\mathcal{T}}$ there is no timed configuration $\langle s, \nu\rangle$ such that $\left\langle\widetilde{s_{0}}, \widetilde{\nu_{0}}\right\rangle \stackrel{d}{\rightarrow}\langle s, \nu\rangle$ and $\left\langle\widetilde{\mu}(s), \widetilde{\eta}^{-1}(\nu)\right\rangle=$ $\left\langle\overline{s_{4}}, \overline{\nu_{0}}\right\rangle$. Then, $(\widetilde{\mu}, \widetilde{\eta})$ is not a $\mathbf{P}_{b i s}$-open morphism, by Proposition 3 . On the other hand, contemplate the timed transition system $\overrightarrow{\mathcal{T}}$ shown in Fig. 4 and timed transition system $\widetilde{\mathcal{T}}$ shown in Fig. 2(d). Define a mapping $\vec{\mu}_{1}: S_{\overrightarrow{\mathcal{T}}} \rightarrow S_{\widetilde{\mathcal{T}}}$ as follows: $\vec{\mu}_{1}\left(\overrightarrow{s_{i, j}}\right)=\widetilde{s}_{i}(0 \leq i \leq 6$, $0 \leq j \leq 4)$, and take the identity mapping $\vec{\eta}_{1}: V_{\widetilde{\mathcal{T}}} \rightarrow V_{\overrightarrow{\mathcal{T}}}$. It is easy to see that $\left(\vec{\mu}_{1}, \vec{\eta}_{1}\right)$ is a tbis-morphism from $\overrightarrow{\mathcal{T}}$ to $\widetilde{\mathcal{T}}$ because $\vec{\mu}_{1}$ and $\vec{\eta}_{1}$ are indeed mappings and the $\left(\vec{\mu}_{l}, \vec{\eta}_{l}\right)$ image of any run in $\overrightarrow{\mathcal{T}}$ is a run in $\tilde{\mathcal{T}}$. Moreover, using Proposition 3, we can conclude that

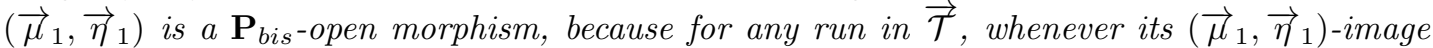
can be extended by an occurrence of some action at some time in $\tilde{\mathcal{T}}$, then the run can also be extended by an occurrence of the same action at same time in $\overrightarrow{\mathcal{T}}$, and the extension in $\widetilde{\mathcal{T}}$ is the $\left(\vec{\mu}_{1}, \vec{\eta}_{1}\right)$-image of the extension in $\overrightarrow{\mathcal{T}}$. For example, treat the run $\gamma=\left\langle\overrightarrow{s_{0,0}}, \overrightarrow{\nu_{0}}\right\rangle \underset{0}{\stackrel{a}{\rightarrow}}\left\langle\overrightarrow{s_{1,1}}, \overrightarrow{\nu_{1}}\right\rangle \stackrel{b}{\rightarrow}$ $\left\langle\overrightarrow{s_{6,2}}, \overrightarrow{\nu_{0}}\right\rangle$ in $\overrightarrow{\mathcal{T}}$. It is not difficult to see that the $\left(\vec{\mu}_{1}, \vec{\eta}_{1}\right)$-image of the run $\gamma$ can be extended by an occurrence of an action c at time 4 up to the run, say, $\widetilde{\gamma}$ in $\widetilde{\mathcal{T}}$, the run $\gamma$ can also be extended by an occurrence of an action c at time 4 up to the run, say, $\vec{\gamma}$ in $\overrightarrow{\mathcal{T}}$, and $\widetilde{\gamma}$ is the $\left(\vec{\mu}_{1}, \vec{\eta}_{1}\right)$-image of the run $\vec{\gamma}$.

Category $\mathbf{T T S} \mathbf{S}_{b b i s}$. This category contains objects which are timed transition systems over an alphabet $\Sigma_{\tau}$ (see Definition 1) and morphisms which represent some notions of simulation of the behavior of one system by the other with an accuracy of $\tau$-actions and with account of only the existence of visible actions. This leads to the following definition of a morphism consisting of two functions, one mapping $\tau$-accessible states of the simulated system to simulating $\tau$ accessible states of the other, and one mapping clocks of the simulating system to simulated clocks. 
Definition 13. Given timed transition systems $\mathcal{T}=\left(S, s_{0}, \quad \Sigma_{\tau}, V, T\right)$ and $\mathcal{T}^{\prime}=$ $\left(S^{\prime}, s_{0}^{\prime}, \Sigma_{\tau}, V^{\prime}, T^{\prime}\right)$, over $\Sigma_{\tau}$, a pair $(\mu, \eta)$ is a tbbis-morphism between $\mathcal{T}$ and $\mathcal{T}^{\prime}$, if $\mu: S_{\tau}(\mathcal{T}) \rightarrow S_{\tau}\left(\mathcal{T}^{\prime}\right)$ is a mapping between the $\tau$-accessible states and $\eta: V^{\prime} \rightarrow V$ is a mapping between clock variables, which must satisfy the following condition: for any run $\left\langle s_{0}, \nu_{0}\right\rangle \underset{d_{1}}{\vec{\tau}}\left\langle s_{1}, \nu_{1}\right\rangle \ldots\left\langle s_{n-1}, \nu_{n-1}\right\rangle \underset{d_{n}}{\stackrel{\tau}{\rightarrow}}\left\langle s_{n}, \nu_{n}\right\rangle$ in $\mathcal{T},\left\langle\mu\left(s_{0}\right), \eta^{-1}\left(\nu_{0}\right)\right\rangle \underset{d_{1}}{\stackrel{\tau}{\rightarrow}}\left\langle\mu\left(s_{1}\right), \eta^{-1}\left(\nu_{1}\right)\right\rangle \ldots$ $\left\langle\mu\left(s_{n-1}\right), \eta^{-1}\left(\nu_{n-1}\right)\right\rangle \underset{d_{n}}{\stackrel{\tau}{\rightarrow}}\left\langle\mu\left(s_{n}\right), \eta^{-1}\left(\nu_{n}\right)\right\rangle$ is a run in $\mathcal{T}^{\prime}$, and whenever $\left\langle s_{n}, \nu_{n}\right\rangle \underset{d}{\vec{\sigma}}(\sigma \in \Sigma)$ in $\mathcal{T}$, then $\left\langle\mu\left(s_{n}\right), \eta^{-1}\left(\nu_{n}\right)\right\rangle \underset{d}{\stackrel{\sigma^{\prime}}{\rightarrow}}\left(\sigma^{\prime} \in \Sigma\right)$ in $\mathcal{T}^{\prime}$.

Example 11. Treat the timed transition systems $\dot{\mathcal{T}}$ and $\ddot{\mathcal{T}}$ shown in Fig. 3. Define a mapping $\dot{\mu}: S_{\dot{\mathcal{T}}} \rightarrow S_{\ddot{\mathcal{T}}}$ as follows: $\dot{\mu}\left(\dot{s}_{2 j}\right)=\ddot{s_{0}}, \dot{\mu}\left(s_{2 j+1}\right)=\ddot{s_{1}}(0 \leq j \leq 1)$, and a mapping $\dot{\eta}: V_{\ddot{\mathcal{T}}} \rightarrow V_{\dot{\mathcal{T}}}$ as follows: $\dot{\eta}(x)=\dot{\eta}(y)=x$. Obviously, $\dot{\mu}$ and $\dot{\eta}$ are indeed mappings. Also, it is easy to see that the $(\dot{\mu}, \dot{\eta})$-images of the runs of $\left(\tau, d_{1}\right) \ldots\left(\tau, d_{n}\right)(n \geq 0)$ in $\dot{\mathcal{T}}$ are runs of the same timed word in $\dot{\mathcal{T}}$, and, moreover, whenever in $\dot{\mathcal{T}}$ the runs can be extended by an occurrence of some action $\sigma \in\{a, b\}$ at some time, then in $\ddot{\mathcal{T}}$ their $(\dot{\mu}, \dot{\eta})$-images can be extended by an occurrence of an action $b$ at same time. For example, the $(\dot{\mu}, \dot{\eta})$-image of the run $\dot{r}=\left\langle\dot{s_{0}}, \dot{\nu_{0}}\right\rangle \stackrel{\tau}{\rightarrow}\left\langle\dot{s_{1}}, \dot{\nu_{0}}\right\rangle$ $\underset{2}{\stackrel{\tau}{\rightarrow}}\left\langle\dot{s_{2}}, \dot{\nu_{0}}\right\rangle$ in $\dot{\mathcal{T}}$ is the run $\ddot{r}=\left\langle\ddot{s_{0}}, \ddot{\nu_{0}}\right\rangle \underset{1}{\stackrel{\tau}{\rightarrow}}\left\langle\ddot{s_{1}}, \ddot{\nu_{0}}\right\rangle \underset{2}{\stackrel{\tau}{\rightarrow}}\left\langle\ddot{s_{0}}, \ddot{\nu_{0}}\right\rangle$ in $\ddot{\mathcal{T}}$. Furthermore, in $\dot{\mathcal{T}} \dot{r}$ can be extended by an occurrence of the action a at time 2 , and in $\ddot{\mathcal{T}} \ddot{r}$ can be extended by an occurrence of the action $b$ at same time. Hence, we may conclude that $(\dot{\mu}, \dot{\eta})$ is a $t_{b b i s}$-morphism.

Timed transition systems over $\Sigma_{\tau}$ and $t_{b b i s}$-morphisms between them form a category of timed transition systems, $\mathbf{T T S}_{b b i s}$, in which the composition of two morphisms $(\mu, \eta): \mathcal{T} \rightarrow \mathcal{T}^{\prime}$ and $\left(\mu^{\prime}, \eta^{\prime}\right): \mathcal{T}^{\prime} \rightarrow \mathcal{T}^{\prime \prime}$ is defined as $\left(\mu^{\prime}, \eta^{\prime}\right) \circ(\mu, \eta):=\left(\mu^{\prime} \circ \mu, \eta \circ \eta^{\prime}\right)$, and the $t_{b b i s}$-morphism is a pair of identity functions.

Following the standards of timed transition systems over $\Sigma_{\tau}$, we construct a subcategory of path objects as follows.

Definition 14. The full subcategory $\mathbf{P}_{\text {bbis }}$ of the category $\mathbf{T} \mathbf{T} \mathbf{S}_{b b i s}$ contains objects $\mathcal{T}^{\alpha,-}=$ $\left(S^{\alpha,-}, 0, \Sigma_{\tau}, V^{\alpha,-}, T^{\alpha,-}\right)$ corresponding to a timed word $\alpha=\left(\tau, d_{1}\right) \ldots\left(\tau, d_{n}\right)$ over $\Sigma_{\tau}$ and a symbol $-\in\{\cdot,(\sigma, d) \mid \sigma \in \Sigma, d \in \mathbf{R}\}$ (the symbol "." denotes "nothing"), where $\mathcal{T}^{\alpha, \cdot}$ is defined as $\mathcal{T}^{\alpha}$ in Definition 12, and $\mathcal{T}^{\alpha,(\sigma, d)}$ is defined as follows: $S^{\alpha,(\sigma, d)}=S^{\alpha, \cdot} \cup\{(n+1)\}$ with the initial state $0, V^{\alpha,(\sigma, d)}=V^{\alpha, \cdot}, T^{\alpha,(\sigma, d)}=T^{\alpha, \cdot} \cup\left\{n \underset{\delta, \emptyset}{\vec{\sigma}}(n+1) \mid \delta=\underset{x \in V^{\alpha,(\sigma, d)}}{\wedge}(x=\right.$ $\left.\left.d-d_{I((n+1), x)}\right)\right\}$, and $t_{b b i s}$-morphisms between the objects.

Lemma 4. Given an object $\mathcal{T}^{\alpha,-}$ in $\mathbf{P}_{\text {bbis }}$ and an object $\mathcal{T}$ in $\mathbf{T} \mathbf{T S}_{b b i s}$,

(i) there is a unique run of $\alpha$ in $\mathcal{T}^{\alpha,-}$ which can be extended by an occurrence of an action $\sigma$ at time $d$, if $-=(\sigma, d)$.

(ii) there is a bijection between the runs $\gamma$ of $\alpha$ in $\mathcal{T}$, which can be extended by an occurrence of an action $\sigma^{\prime} \in \Sigma$ at time $d$, if $-=(\sigma, d)$, and the $t_{b b i s}$-morphisms $(\mu, \eta): \mathcal{T}^{\alpha,-} \rightarrow \mathcal{T}$ such that the runs $\gamma$ are the $(\mu, \eta)$-images of the run of $\alpha$ in $\mathcal{T}^{\alpha,-}$.

Proposition 4. Given objects $\mathcal{T}, \mathcal{T}^{\prime}$ in $\mathbf{T T S}_{\text {bbis }}$, a morphism $(\mu, \eta): \mathcal{T} \longrightarrow \mathcal{T}^{\prime}$ in $\mathbf{T T S}_{\text {bbis }}$ is $\mathbf{P}_{\text {bbis }}$-open iff for any run $\left\langle s_{0}, \nu_{0}\right\rangle \underset{d_{1}}{\vec{\tau}}\left\langle s_{1}, \nu_{1}\right\rangle \ldots\left\langle s_{n-1}, \nu_{n-1}\right\rangle \underset{d_{n}}{\stackrel{\tau}{\rightarrow}}\left\langle s_{n}, \nu_{n}\right\rangle$ in $\mathcal{T}$,

- whenever $\left\langle\mu\left(s_{n}\right), \eta^{-1}\left(\nu_{n}\right)\right\rangle \underset{d}{\vec{\tau}}\left\langle s^{\prime}, \nu^{\prime}\right\rangle$ in $\mathcal{T}^{\prime}$, then $\left\langle s_{n}, \nu_{n}\right\rangle \stackrel{\tau}{\vec{d}}\langle s, \nu\rangle$ in $\mathcal{T}$ and $\left\langle\mu(s), \eta^{-1}(\nu)\right\rangle$ $=\left\langle s^{\prime}, \nu^{\prime}\right\rangle$, 


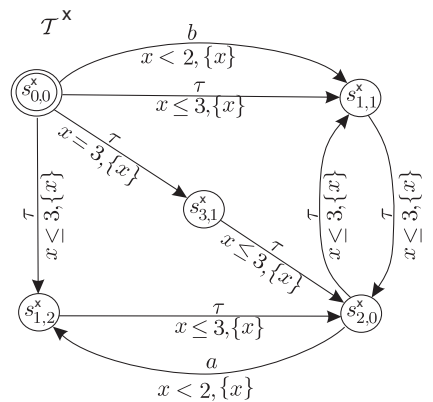

Figure 5: An example of a vertex of span between timed transition systems

- whenever $\left\langle\mu\left(s_{n}\right), \eta^{-1}\left(\nu_{n}\right)\right\rangle \underset{d}{\stackrel{\sigma}{\vec{d}}}(\sigma \in \Sigma)$ in $\mathcal{T}^{\prime}$, then $\left\langle s_{n}, \nu_{n}\right\rangle \underset{d}{\stackrel{\sigma^{\prime}}{\rightarrow}}\left(\sigma^{\prime} \in \Sigma\right)$ in $\mathcal{T}$.

Example 12. Consider the systems $\dot{\mathcal{T}}$ and $\ddot{\mathcal{T}}$ shown in Fig. 3, and the $t_{b b i s}$-morphism $(\dot{\mu}, \dot{\eta})$ from $\dot{\mathcal{T}}$ to $\ddot{\mathcal{T}}$, specified in Example 11 . Clearly, the run $\ddot{\gamma}=\left\langle\ddot{s}_{0}, \ddot{\nu_{0}}\right\rangle$ in $\ddot{\mathcal{T}}$ is the $(\dot{\mu}, \dot{\eta})$-image of $\dot{\gamma}=\left\langle\dot{s_{0}}, \dot{\nu}_{0}\right\rangle$ in $\dot{\mathcal{T}}$. Moreover, $\ddot{\gamma}$ can be extended by an occurrence of an action $\tau$ at time 0 up to the run $\ddot{\gamma}_{1}=\left\langle\ddot{s_{0}}, \ddot{\nu_{0}}\right\rangle \underset{0}{\stackrel{\tau}{\rightarrow}}\left\langle\ddot{s_{2}}, \ddot{\nu_{0}}\right\rangle$ but in $\dot{\mathcal{T}}$ there is no timed configuration $\langle s, \nu\rangle$ such that $\left\langle\dot{\mu}(s), \dot{\eta}^{-1}(\nu)\right\rangle=\left\langle\ddot{s_{2}}, \ddot{\nu_{0}}\right\rangle$. Hence, the $t_{b b i s}$-morphism $(\dot{\mu}, \dot{\eta})$ is not $\mathbf{P}_{\text {bbis }}$-open, by Proposition 4 . Further, contemplate the system $\mathcal{T}^{\times}$shown in Fig. 5. Define a mapping $\mu_{1}^{\times}: S_{\tau}^{\mathcal{T}^{\times}} \rightarrow S_{\tau}^{\dot{\mathcal{T}}}$ as follows: $\mu_{1}^{\times}\left(s_{i, j}^{\times}\right)=\dot{s}_{i}(0 \leq i \leq 3,0 \leq j \leq 2)$, and take the identity function $\eta_{1}^{\times}: V_{\dot{\mathcal{T}}} \rightarrow V_{\mathcal{T}^{\times}}$. Clearly, $\mu_{1}^{\times}$and $\eta_{1}^{\times}$are indeed mappings. Moreover, it is easy to see that the $\left(\mu_{1}^{\times}, \eta_{1}^{\times}\right)$-images of the runs of $\left(\tau, d_{1}\right) \ldots\left(\tau, d_{n}\right)(n \geq 0)$ in $\mathcal{T}^{\times}$are also runs of the same timed word in $\dot{\mathcal{T}}$, and whenever in $\mathcal{T}^{\times}$a run of $\left(\tau, d_{1}\right) \ldots\left(\tau, d_{n}\right)(n \geq 0)$ can be extended by an occurrence of a non- $\tau$-action at some time, then in $\dot{\mathcal{T}}$ its $\left(\mu_{1}^{\times}, \eta_{1}^{\times}\right)$-image can also be extended by an occurrence of a non- $\tau$-action at same time. This means that $\left(\mu_{1}^{\times}, \eta_{1}^{\times}\right)$is a $t_{b b i s}$-morphism. Furthermore,

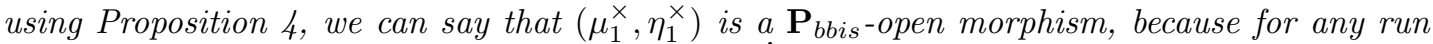
of $\left(\tau, d_{1}\right) \ldots\left(\tau, d_{n}\right)(n \geq 0)$ in $\mathcal{T}^{\times}$, whenever in $\dot{\mathcal{T}}$ its $\left(\mu_{1}^{\times}, \eta_{1}^{\times}\right)$-image can be extended by an occurrence of some action at some time, then in $\mathcal{T}^{\times}$the run can be extended by an occurrence of the same action at same time. For instance, treat the run $\gamma^{\times}=\left\langle s_{0,0}^{\times}, \nu_{0,0}^{\times}\right\rangle \underset{1}{\stackrel{\tau}{\rightarrow}}\left\langle s_{3,0}^{\times}, \nu_{0,0}^{\times}\right\rangle \underset{2}{\stackrel{\tau}{\rightarrow}}$ $\left\langle s_{2,0}^{\times}, \nu_{0,0}^{\times}\right\rangle$in $\mathcal{T}^{\times}$. It is not difficult to see that in $\dot{\mathcal{T}}$ the $\left(\mu_{1}^{\times}, \eta_{1}^{\times}\right)$-image of the run $\gamma^{\times}$can be extended by an occurrence of the action a at time 3 , and in $\mathcal{T}^{\times}$the run $\gamma^{\times}$can also be extended by an occurrence of the action a at time 3.

Finally, for $* \in\{$ trace, test, bis, bbis $\}$, the coincidence of $\mathbf{P}_{*}$-bisimilarity and $*$-equivalence is established.

Theorem 1. Let $* \in\{$ trace, test, bis, bbis $\}$. Timed transition systems from $\mathbf{T} \mathbf{T S} \mathbf{S}_{*}$ are $\mathbf{P}_{*-}$ bisimilar iff they are $*$-equivalent.

Example 13. First, contemplate the systems $\mathcal{T}^{\prime}$ and $\mathcal{T}^{\prime \prime}$ depicted in Fig. $2(b)$. From Example 1 .

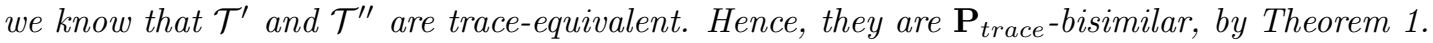

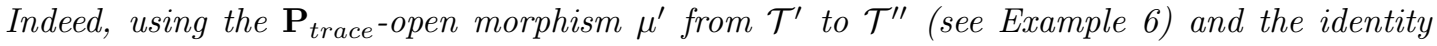

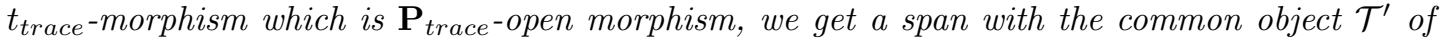
the $\mathbf{P}_{\text {trace-open morphisms. }}$

Second, examine the systems $\widehat{\mathcal{T}}$ and $\check{\mathcal{T}}$ depicted in Fig. 2(c). As shown in Example 2, $\widehat{\mathcal{T}}$ and

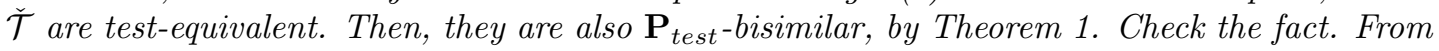




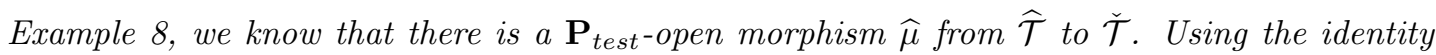

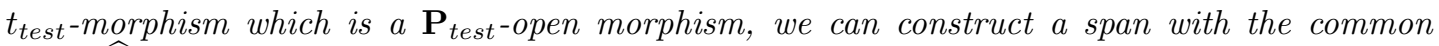
object $\widehat{\mathcal{T}}$ of the $\mathbf{P}_{\text {test }}$-open morphisms.

Third, treat the systems $\widetilde{\mathcal{T}}$ and $\overline{\mathcal{T}}$ depicted in Fig. $2($ d). As demonstrated in Example $3 . \widetilde{\mathcal{T}}$ and $\overline{\mathcal{T}}$ are bis-equivalent. Hence, these systems are $\mathbf{P}_{\text {bis }}$-bisimilar, according to Theorem 1. To verify the fact, we need the timed transition system $\overrightarrow{\mathcal{T}}$ depicted in Fig. 4. Define a mapping $\vec{\mu}_{2}: S_{\overrightarrow{\mathcal{T}}} \rightarrow S_{\overline{\mathcal{T}}}$ as follows: $\vec{\mu}_{2}\left(\overrightarrow{s_{i, j}}\right)=\overline{s_{j}}$, for all $0 \leq i \leq 6$ and $0 \leq j \leq 4$, and take the identity mapping $\vec{\eta}_{2}: V_{\overline{\mathcal{T}}} \rightarrow V_{\overrightarrow{\mathcal{T}}}$. It is easy to see that $\left(\vec{\mu}_{2}, \vec{\eta}_{2}\right)$ is a $t_{\text {bis }}$-morphism, and, moreover,

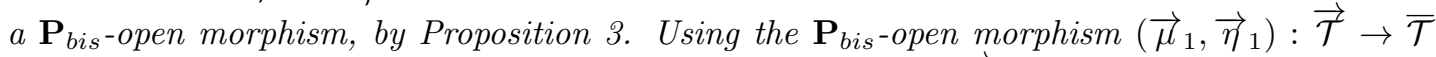
specified in Example 10, we get a span with the common object $\overrightarrow{\mathcal{T}}$ of the $\mathbf{P}_{\text {bis }}$-open morphisms.

Finally, consider the bbis-equivalent systems $\dot{\mathcal{T}}$ and $\ddot{\mathcal{T}}$ shown in Fig. 3 (see Example 4 ). Due to Theorem 1 . $\dot{\mathcal{T}}$ and $\ddot{\mathcal{T}}$ are $\mathbf{P}_{\text {bis }}$-bisimilar. Contemplate the system $\mathcal{T}^{\times}$depicted in Fig. 5 and the $\mathbf{P}_{\text {bbis }}$-open morphism $\left(\mu_{1}^{\times}, \eta_{1}^{\times}\right)$from $\mathcal{T}^{\times}$to $\mathcal{T}$, specified in Example 12. Further, define a mapping $\mu_{2}^{\times}: S_{\tau}^{\mathcal{T}^{\times}} \rightarrow S_{\tau}^{\ddot{\mathcal{T}}}$ as follows: $\mu_{2}^{\times}\left(s_{i, j}^{\times}\right)=\dot{s_{j}}(0 \leq i \leq 3,0 \leq j \leq 2)$, and a mapping $\eta_{2}^{\times}: V_{\ddot{\mathcal{T}}} \rightarrow V_{\mathcal{T} \times}$ as follows: $\eta_{2}^{\times}(x)=\eta_{2}^{\times}(y)=x$. Using Proposition 4, it is easy to check that $\left(\mu_{2}^{\times}, \eta_{2}^{\times}\right)$is a $\mathbf{P}_{\text {bbis }}$-open $t_{b b i s}-$ morphism from $\mathcal{T}^{\times}$to $\ddot{\mathcal{T}}$. Thus, we have a span with the common object $\mathcal{T}^{\times}$of the $\mathbf{P}_{\text {bbis }}$-open morphisms.

\section{Path-Bisimulation}

To obtain a logic characteristic of bisimulation induced by open maps, Joyal, Nielsen, and Winskel [14 have proposed a second category-theoretic characterization of bisimulation path bisimulation which is a relation based generalization of open maps bisimulation.

Definition 15. Let $\mathbb{M}$ be a category of models, let $\mathbb{P}$ be a small category of path objects, where $\mathbb{P}$ is a subcategory of $\mathbb{M}$, let $I$ be a common initial object ${ }^{4}$ in $\mathbb{P}$ and $\mathbb{M}$. Then,

- Two objects $X_{1}$ and $X_{2}$ in $\mathbb{M}$ are called path-P-bisimilar iff there is a set $\mathcal{R}$ of pairs of paths $\left(p_{1}, p_{2}\right)$ with common domain $P$, so $p_{1}: P \rightarrow X_{1}$ is a path in $X_{1}$ and $p_{2}: P \rightarrow X_{2}$ is a path in $X_{2}$, such that

(o) $\left(i_{1}, i_{2}\right) \in \mathcal{R}$, where $i_{1}: I \rightarrow X_{1}$ and $i_{2}: I \rightarrow X_{2}$ are the unique paths starting in the initial object, and for all $\left(p_{1}, p_{2}\right) \in \mathcal{R}$ and for all $m: P \rightarrow Q$, where $m$ is in $\mathbb{P}$, holds

(i) if there exists $q_{1}: Q \rightarrow X_{1}$ with $q_{1} \circ m=p_{1}$ then there exists $q_{2}: Q \rightarrow X_{2}$ with $q_{2} \circ m=p_{2}$ and $\left(q_{1}, q_{2}\right) \in \mathcal{R}$ and

(ii) if there exists $q_{2}: Q \rightarrow X_{2}$ with $q_{2} \circ m=p_{2}$ then there exists $q_{1}: Q \rightarrow X_{1}$ with $q_{1} \circ m=p_{1}$ and $\left(q_{1}, q_{2}\right) \in \mathcal{R}$.

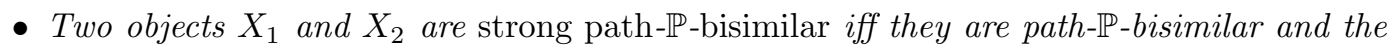
set $\mathcal{R}$ further satisfies:

(iii) If $\left(q_{1}, q_{2}\right) \in \mathcal{R}$, with $q_{1}: Q \rightarrow X_{1}$ and $q_{2}: Q \rightarrow X_{2}$ and $m: P \rightarrow Q$, where $m$ is in $\mathbb{P}$, then $\left(q_{1} \circ m, q_{2} \circ m\right) \in \mathcal{R}$.

Consider an auxiliary fact for the cases $* \in\{$ trace, test $\}$.

\footnotetext{
${ }^{4}$ In the cases when $\mathbb{P}$ is $\mathbf{P}_{*}$ and $\mathbb{M}$ is $\mathbf{T}$ TS $\mathbf{S}_{*}$, the initial object $I_{*}$ is the timed transition system $\left(\left\{s_{0}\right\}, s_{0}, \Sigma,\{x\}, \emptyset\right)$ for $* \in\{$ trace, test, bis $\}$, and $\left(\left\{s_{0}\right\}, s_{0}, \Sigma_{\tau},\{x\}, \emptyset\right)$ for $*=$ bbis.
} 
Lemma 5. Let $\mathcal{R}$ be a path- $\mathbf{P}_{*}$-bisimulation between $\mathcal{T}_{1}$ and $\mathcal{T}_{2}$ and $* \in\{$ trace, test $\}$. Given $\alpha \in \mathcal{L}\left(\mathcal{T}_{i}\right)$ for some $i=1,2$, there exists a pair $\left(p_{1}, p_{2}\right) \in \mathcal{R}$ such that $p_{j}: P \rightarrow \mathcal{T}_{j}$ for all $j=1,2$, where $P=\mathcal{T}^{\alpha}$, if $*=$ trace, and $P=\mathcal{T}^{\alpha, \emptyset}$, otherwise.

We are now ready to establish the main result of this section.

Theorem 2. For $* \in\{$ trace, test, bis, bbis $\}, \mathbf{P}_{*}$-bisimilarity, path- $\mathbf{P}_{*}$-bisimilarity, strong path$\mathbf{P}_{*}$-bisimilarity all coincide with $*$-equivalence.

Example 14. To illustrate the meaning of the theorem, consider the most interesting case, i.e. the test-equivalent systems $\widehat{\mathcal{T}}$ and $\breve{\mathcal{T}}$ from Example 2. According to Theorem 2, they are path-

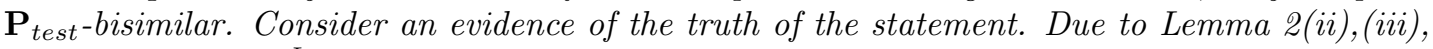
for any object $\mathcal{T}^{\alpha, L}$ in $\mathbf{P}_{\text {test }}$ and any object $\mathcal{T}$ in $\mathbf{T} \mathbf{T} \mathbf{S}_{\text {test }}$, there is a bijection between the

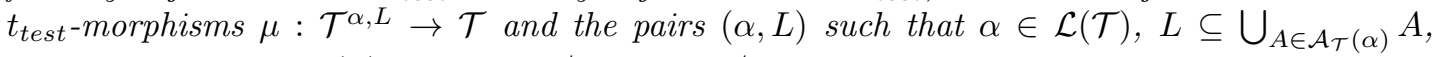
and for all $A \in \mathcal{A}_{\mathcal{T}}(\alpha) A \cap L \neq \emptyset$, if $L \neq \emptyset$. Also, we know that there is at most one $t_{\text {test }}$-morphism between timed transition systems. Specify a relation $\widehat{\mathcal{R}}=\widehat{\mathcal{R}}_{1} \cup \widehat{\mathcal{R}}_{2}$, where $\widehat{\mathcal{R}}_{1}=\left\{\left(p_{\widehat{\mathcal{T}}}^{\varepsilon,\{(a, d)\}}: \mathcal{T}^{\varepsilon,\{(a, d)\}} \rightarrow \widehat{\mathcal{T}}, p_{\tilde{\mathcal{T}}}^{\varepsilon,\{(a, d)\}}: \mathcal{T}^{\varepsilon,\{(a, d)\}} \rightarrow \check{\mathcal{T}}\right) \mid d \leq 1\right\}$ and $\widehat{\mathcal{R}}_{2}=\left\{\left(p_{\widehat{\mathcal{T}}}^{\alpha, \emptyset}:\right.\right.$ $\left.\left.\mathcal{T}^{\alpha, \emptyset} \rightarrow \widehat{\mathcal{T}}, p_{\tilde{\mathcal{T}}}^{\alpha, \emptyset}: \mathcal{T}^{\alpha, \emptyset} \rightarrow \check{\mathcal{T}}\right) \mid \alpha \in \mathcal{L}(\widehat{\mathcal{T}})=\mathcal{L}(\check{\mathcal{T}})\right\}$. Obviously, $\left(p_{\widehat{\mathcal{T}}}^{\varepsilon, \emptyset}, p_{\tilde{\mathcal{T}}}^{\varepsilon, \emptyset}\right) \in \widehat{\mathcal{R}}$. Take arbitrary $\left(p_{\widehat{\mathcal{T}}}^{\alpha, L}, p_{\tilde{\mathcal{T}}}^{\alpha, L}\right) \in \widehat{\mathcal{R}}$ and $m: \mathcal{T}^{\alpha, L} \rightarrow \mathcal{T}^{\alpha^{\prime}, L^{\prime}}$ in $\mathbf{P}_{\text {test. }}$. Suppose that there is $p_{\widehat{\mathcal{T}}}^{\alpha^{\prime}, L^{\prime}}: \mathcal{T}^{\alpha^{\prime}, L^{\prime}} \rightarrow \widehat{\mathcal{T}}$ such that $p_{\widehat{\mathcal{T}}}^{\alpha, L}=p_{\widehat{\mathcal{T}}}^{\alpha^{\prime}, L^{\prime}} \circ m$. (The case when there is $p_{\check{\mathcal{T}}}^{\alpha^{\prime}, L^{\prime}}: \mathcal{T}^{\alpha^{\prime}, L^{\prime}} \rightarrow \check{\mathcal{T}}$ such that $p_{\breve{\mathcal{T}}}^{\alpha, L}=p_{\breve{\mathcal{T}}}^{\alpha^{\prime}, L^{\prime}} \circ m$ is similar.) From example 2, we know that the systems $\widehat{\mathcal{T}}$ and $\check{\mathcal{T}}$ have the same languages, and in both the systems, there is the only run of the empty timed word which can be extended only by an occurrence of an action a at time $d$, where $d \leq 1$, and, moreover, for any non-empty timed word from the systems' languages there is the run of the timed word, which cannot be extended. This means that for any $t_{\text {test }}$-morphism $p_{\mathcal{T}}^{\alpha, L}: \mathcal{T}^{\alpha, L} \rightarrow \mathcal{T} \in\{\widehat{\mathcal{T}}, \check{\mathcal{T}}\}$, if $\alpha \neq \varepsilon$, then $L=\emptyset$, and if $\alpha=\varepsilon$, then $L \subseteq \bigcup_{A \in \mathcal{A}_{\mathcal{T}}(\varepsilon)=\{A\}} A=\{(a, d) \mid(a, d) \in \mathcal{L}(\mathcal{T})\}$, and for all $A \in \mathcal{A}_{\mathcal{T}}(\varepsilon)$ $A \cap L \neq \emptyset$, if $L \neq \emptyset$. Hence, there exists $p_{\check{\mathcal{T}}}^{\alpha^{\prime}, L^{\prime}}: \mathcal{T}^{\alpha^{\prime}, L^{\prime}} \rightarrow \check{\mathcal{T}}$ such that $p_{\check{\mathcal{T}}}^{\alpha, L}=p_{\check{\mathcal{T}}}^{\alpha^{\prime}, L^{\prime}} \circ m$ and $\left(p_{\widehat{\mathcal{T}}}^{\alpha^{\prime}, L^{\prime}}, p_{\tilde{\mathcal{T}}}^{\alpha^{\prime}, L^{\prime}}\right) \in \widehat{\mathcal{R}}$. Thus, $\widehat{\mathcal{R}}$ is indeed a path-P $\mathbf{P}_{\text {test }}$-bisimulation.

\section{Coalgebraic Bisimulation}

Another alternative abstract characterization of bisimulation is based on a category of coalgebras induced by an endofunctor on an arbitrary category. In [16] it has been shown that the concept of path-bisimilarity can be translated into a coalgebraic setting with lax cohomomorphisms. Notice, in [22] a coalgebraic characterization of path-bisimilarity is obtained without the use of lax notions, however, in this case one cannot define a functor from a category of computations to the category of coalgebras.

We start with defining the terminology from [16. Let $\mathbb{M}$ be a locally small category with a small path subcategory $\mathbb{P}$. We will define an embedding of $\mathbb{M}$ into a category of coalgebras for some endofunctor on the category $S e t^{|\mathbb{P}|}$ of $|\mathbb{P}|$-sorted sets $(|\mathbb{P}|$-indexed sets), where $|\mathbb{P}|$ is the set of objects in $\mathbb{P}$. The endofunctor $F_{\mathbb{P}}:$ Set $t^{|\mathbb{P}|} \longrightarrow S e t^{|\mathbb{P}|}$ is defined as follows:

$$
\left\{X_{P}\right\}_{P \in|\mathbb{P}|} \longmapsto\left\{\prod_{Q \in|\mathbb{P}|}\left(\mathcal{P}\left(X_{Q}\right)\right)^{\text {Hom }_{\mathbb{P}}(P, Q)}\right\}_{P \in|\mathbb{P}|},
$$

where $\mathcal{P}(\cdot)$ denotes the powerset, $X_{P}$ specifies a component of a $|\mathbb{P}|$-sorted set $X$ for $P \in|\mathbb{P}|$, and $\operatorname{Hom}_{\mathbb{P}}(P, Q)$ stands for the set of all morphisms from $P$ to $Q$ in $\mathbb{P}$. On morphisms in the 
category $S e t^{|\mathbb{P}|}$, the endofunctor $F_{\mathbb{P}}$ acts by the following rule:

$$
F_{\mathbb{P}}:\left(\left\{\gamma_{P}\right\}_{P \in|\mathbb{P}|}: X \rightarrow Y\right) \longmapsto\left\{\prod_{Q \in|\mathbb{P}|} h_{Q}^{P}\right\}_{P \in|\mathbb{P}|},
$$

where $h_{Q}^{P}: \mathcal{P}\left(X_{Q}\right)^{\operatorname{Hom}_{\mathbb{P}}(P, Q)} \rightarrow \mathcal{P}\left(Y_{Q}\right)^{\operatorname{Hom}_{\mathbb{P}}(P, Q)}: g \longmapsto f, f(m)=\left\{\gamma_{Q}(x) \mid x \in g(m)\right\}$ for all $m \in \operatorname{Hom}_{\mathbb{P}}(P, Q)$.

A coalgebra for $F_{\mathbb{P}}$ or $F_{\mathbb{P}}$-coalgebra is a pair $(S, t r)$ with $S$ an object in $S e t^{|\mathbb{P}|}$ and $t r: S \rightarrow$ $F_{\mathbb{P}}(S)$ a morphism in $S e t^{|\mathbb{P}|}$, which consists of a family of functions:

$$
\left\{\operatorname{tr}_{P}: S_{P} \rightarrow \prod_{Q \in|\mathbb{P}|}\left(\mathcal{P}\left(S_{Q}\right)\right)^{\operatorname{Hom}_{\mathbb{P}}(P, Q)}\right\}_{P \in|\mathbb{P}|} \cdot
$$

The set $S$ is called the carrier and the function $t r$ is called the coalgebra structure of the

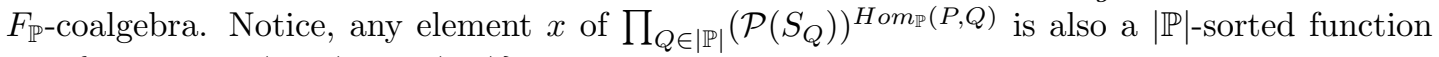
$x=\left\{x_{Q}: \operatorname{Hom}_{\mathbb{P}}(P, Q) \rightarrow \mathcal{P}\left(S_{Q}\right)\right\}_{Q \in|\mathbb{P}|}$. Due to the canonical bijection

$$
\prod_{Q \in|\mathbb{P}|}\left(\mathcal{P}\left(S_{Q}\right)\right)^{\operatorname{Hom}_{\mathbb{P}}(P, Q)} \cong \prod_{m \in \uplus_{Q} \in \mathbb{P} \mid} \prod_{\operatorname{Hom}_{\mathbb{P}}(P, Q)} \mathcal{P}\left(S_{\text {codomain }(m)}\right),
$$

we can omit the subscript and write $x(m)$ for a morphism $m: P \rightarrow Q$ in $\mathbb{P}$ instead of $x_{Q}(m)$ and may represent a coalgebra structure as the following family of functions:

$$
\left\{\operatorname{tr}_{P}: S_{P} \rightarrow \prod_{m \in \uplus_{Q \in|\mathbb{P}|} \operatorname{Hom}_{\mathbb{P}}(P, Q)} \mathcal{P}\left(S_{\operatorname{codomain}(m)}\right)\right\}_{P \in|\mathbb{P}|} .
$$

A morphism $\gamma: S_{1} \rightarrow S_{2}$ in the category $S e t^{|\mathbb{P}|}$ is called a cohomomorphism between $F_{\mathbb{P}^{-}}$ coalgebras $\left(S_{1}, t r_{1}\right)$ and $\left(S_{2}, t r_{2}\right)$ iff $F_{\mathbb{P}}(\gamma) \circ t r_{1}=t r_{2} \circ \gamma . F_{\mathbb{P}}$-coalgebras and cohomomorphisms between them constitute a category, denoted by $\mathcal{C} \mathcal{A}_{\mathbb{P}}$.

From now on, for an $F_{\mathbb{P}^{-}}$coalgebra $(S, t r)$, a triple $\left\langle m_{1}, m, m_{2}\right\rangle$, where $m_{1} \in S_{P}, m_{2} \in S_{Q}$ and $m \in \operatorname{Hom}_{\mathbb{P}}(P, Q)$, satisfying $m_{2} \in \operatorname{tr}_{P}\left(m_{1}\right)(m)$, will be denoted by $m_{1} \stackrel{m}{\rightarrow} m_{2}$.

As usual in the theory of coalgebras, bisimulation is a relation represented by a span of coalgebra morphisms [23]. An $F_{\mathbb{P}^{-}}$bisimulation between two coalgebras $\left(S_{1}, t r_{1}\right)$ and $\left(S_{2}, t r_{2}\right)$ is a $|\mathbb{P}|$-sorted relation $R=\left\{R_{P}\right\}_{P \in|\mathbb{P}|} \subseteq\left(S_{1} \times S_{2}\right)$ such that, if $\left(m_{1}, m_{2}\right) \in R_{P}$ and $m: P \rightarrow Q$ in $\mathbb{P}$, then

- if $m_{1} \stackrel{m}{\rightarrow} m_{1}^{\prime}$, then $m_{2} \stackrel{m}{\rightarrow} m_{2}^{\prime}$ and $\left(m_{1}^{\prime}, m_{2}^{\prime}\right) \in R_{Q}$ for some $m_{2}^{\prime} \in S_{2}$,

- if $m_{2} \stackrel{m}{\rightarrow} m_{2}^{\prime}$, then $m_{1} \stackrel{m}{\rightarrow} m_{1}^{\prime}$ and $\left(m_{1}^{\prime}, m_{2}^{\prime}\right) \in R_{Q}$ for some $m_{1}^{\prime} \in S_{1}$.

Clearly, each $F_{\mathbb{P}}$-bisimulation has a coalgebra structure $\operatorname{tr}_{R}: R \rightarrow F_{\mathbb{P}}(R)$ and together with the projections $\pi_{1}: R \rightarrow S_{1}$ and $\pi_{2}: R \rightarrow S_{2}$ form a span of cohomomorphisms of the $F_{\mathbb{P}}$-coalgebra.

Next, following [16, we relax the requirement on coalgebra morphism. A morphism $\gamma: S \rightarrow$ $S^{\prime}$ in $S e t^{|\mathbb{P}|}$ is called a lax cohomomorphism between $F_{\mathbb{P}}$-coalgebras $(S, t r)$ and $\left(S^{\prime}, t r^{\prime}\right)$ if for each $s \in S_{P}$ and $m \in \operatorname{Hom}_{\mathbb{P}}(P, Q),\left\{\gamma_{Q}(r) \mid r \in \operatorname{tr}_{P}(s)(m)\right\} \subseteq \operatorname{tr}_{P}^{\prime}\left(\gamma_{P}(s)\right)(m) . F_{\mathbb{P}^{-} \text {-coalgebras }}$ and lax cohomomorphisms constitute a category, denoted by $\mathcal{C} \mathcal{A}_{\mathbb{P}}^{\text {lax }}$ (the category $\mathcal{C} \mathcal{A}_{\mathbb{P}}$ contains those lax cohomomorphisms for which the above inclusion is replaced by equality).

For $\mathbb{M}$ with $\mathbb{P}$, define a functor $\mathcal{B}$ eh $h_{\mathbb{P}}^{\mathbb{M}}: \mathbb{M} \rightarrow \mathcal{C} \mathcal{A}_{\mathbb{P}}^{\text {lax }}$. $\mathcal{B}$ eh $h_{\mathbb{P}}^{\mathbb{M}}$ acts on objects $X$ in $\mathbb{M}$ as follows: $\left\{\operatorname{Hom}_{\mathbb{M}}(P, X)\right\}_{P \in|\mathbb{P}|}$ is the carrier and $\left\{\operatorname{tr}_{P}: m_{1} \longmapsto \prod_{m \in \uplus_{Q \in|\mathbb{P}|} H o m_{\mathbb{P}}(P, Q)}\left\{m_{2} \mid m_{1}=\right.\right.$ $\left.\left.m_{2} \circ m\right\}\right\}_{P \in|\mathbb{P}|}$ is the coalgebra structure of the corresponding $F_{\mathbb{P}}$-coalgebra. $\mathcal{B} e h_{\mathbb{P}}^{\mathbb{M}}$ acts on morphisms $f: X \rightarrow Y$ in $\mathbb{M}$ as follows: $\mathcal{B e h}_{\mathbb{P}}^{\mathbb{M}}(f)_{P}: \operatorname{Hom}_{\mathbb{M}}(P, X) \rightarrow \operatorname{Hom}_{\mathbb{M}}(P, Y): \alpha \longmapsto f \circ \alpha$. 
Proposition 5. [16] For any two objects $X$ and $Y$ in $\mathbb{M}, a|\mathbb{P}|$-sorted relation $R$ is a path$\mathbb{P}$-bisimulation between $X$ and $Y$ iff it is an $F_{\mathbb{P}}$-bisimulation between $\mathcal{B e h}_{\mathbb{P}}^{\mathbb{M}}(X)$ and $\mathcal{B e h}_{\mathbb{P}}^{\mathbb{M}}(Y)$ containing the pair $\left(i_{X}, i_{Y}\right)$, where $i_{X}: I \rightarrow X$ and $i_{Y}: I \rightarrow Y$ are paths, with an initial object $I$.

Example 15. In order to elucidate Proposition 5, contemplate the path- $\mathbf{P}_{\text {test }}$-bisimulation $\widehat{\mathcal{R}}$ between the systems $\widehat{\mathcal{T}}$ and $\check{\mathcal{T}}$ from Example 14 . According to the proposition, $\widehat{\mathcal{R}}$ is an $F_{\mathbf{P}_{\text {test }}}$ -

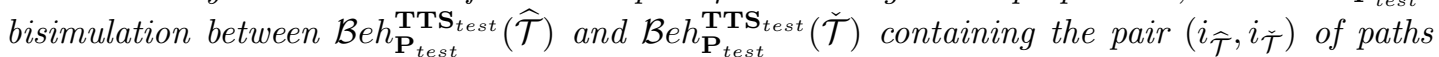
with the initial object $I_{\text {test }}$. Verify this statement. The functor $\mathcal{B}$ eh PTs $_{\mathbf{P}_{\text {test }}}^{\mathbf{T T}_{\text {test }}}$ maps a system $\mathcal{T}$ to the $F_{\mathbf{P}_{\text {test }} \text {-coalgebra with the carrier }}\left\{S_{\mathcal{T}^{\alpha, L}}=\left\{p_{\mathcal{T}}^{\alpha, L} \in \operatorname{Hom}_{\mathbf{T}^{\mathbf{T}} \mathbf{S}_{\text {test }}}\left(\mathcal{T}^{\alpha, L}, \mathcal{T}\right)\right\}\right\}_{\mathcal{T}^{\alpha, L} \in\left|\mathbf{P}_{\text {test }}\right|}$

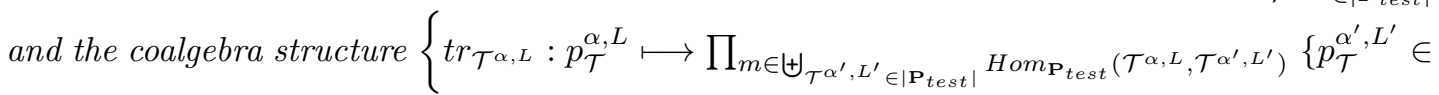
$\left.\left.S_{\mathcal{T}^{\alpha^{\prime}, L^{\prime}}} \mid p_{\mathcal{T}}^{\alpha, L}=p_{\mathcal{T}}^{\alpha^{\prime}, L^{\prime}} \circ m\right\}\right\}_{\mathcal{T}^{\alpha, L} \in\left|\mathbf{P}_{\text {test }}\right|}$. Taking into consideration the minimality of $\mathcal{S} \mathcal{R}(\cdot)$, we have the carrier $\left\{S_{\mathcal{T}^{\alpha, L}}=\left\{p_{\mathcal{T}}^{\alpha, L}\right\}\right\}_{\mathcal{T}^{\alpha, L} \in\left|\mathbf{P}_{\text {trace }}\right|}$ and the coalgebra structure $\left\{\operatorname{tr}_{\mathcal{T}^{\alpha, L}}: p_{\mathcal{T}}^{\alpha, L} \longmapsto\right.$ $\left.\prod_{m \in \biguplus_{\mathcal{T}^{\alpha^{\prime}, L^{\prime}} \in\left|\mathbf{P}_{\text {test }}\right|} \text { Hom }_{\mathbf{P}_{\text {test }}}\left(\mathcal{T}^{\alpha, L}, \mathcal{T}^{\left.\alpha^{\prime}, L^{\prime}\right)}\right.} S_{\mathcal{T}^{\alpha^{\prime}, L^{\prime}}}\right\}_{\mathcal{T}^{\alpha, L} \in\left|\mathbf{P}_{\text {test }}\right|}$. In informal words, the carrier is indexed by 'trees' whose trunks correspond to timed words over $\Sigma$ and whose branches are constructed of sets of actions from $\Sigma$ with times at which the actions occur after executions of the timed words, and the coalgebra structure is marked by all possible mappings from trees to another ones, embedding their trunks and finding in the trees subbranches of the branches of the images of the trees. We know that $\widehat{\mathcal{R}}$ contains $\left(i_{\widehat{\mathcal{T}}}, i_{\breve{\mathcal{T}}}\right)$. Let $\left(p_{\widehat{\mathcal{T}}}^{\alpha, L}, p_{\tilde{\mathcal{T}}}^{\alpha, L}\right) \in \widehat{\mathcal{R}}$. Assume $p_{\widehat{\mathcal{T}}}^{\alpha, L} \stackrel{m}{\rightarrow} p_{\tilde{\mathcal{T}}}^{\alpha^{\prime}, L^{\prime}}$, i.e.

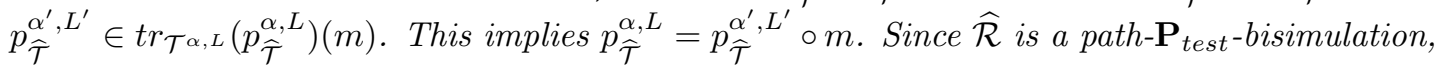
there is $p_{\tilde{\mathcal{T}}}^{\alpha^{\prime}, L^{\prime}}: \mathcal{T}^{\alpha^{\prime}, L^{\prime}} \rightarrow \check{\mathcal{T}}$ such that $p_{\tilde{\mathcal{T}}}^{\alpha, L}=p_{\tilde{\mathcal{T}}}^{\alpha^{\prime}, L^{\prime}} \circ m$ and $\left(p_{\widehat{\mathcal{T}}}^{\alpha^{\prime}, L^{\prime}}, p_{\tilde{\mathcal{T}}}^{\alpha^{\prime}, L^{\prime}}\right) \in \widehat{\mathcal{R}}$. This means $p_{\mathcal{\mathcal { T }}}^{\alpha^{\prime}, L^{\prime}} \in \operatorname{tr}_{\mathcal{T}^{\alpha, L}}\left(p_{\mathcal{\mathcal { T }}}^{\alpha, L}\right)(m)$, i.e. $p_{\mathcal{\mathcal { T }}}^{\alpha, L} \stackrel{m}{\rightarrow} p_{\mathcal{\mathcal { T }}}^{\alpha^{\prime}, L^{\prime}}$. Hence, $\widehat{\mathcal{R}}$ is indeed an $F_{\mathbf{P}_{\text {test }}}$-bisimulation.

Corollary 1. For $* \in\{$ trace, test, bis, bbis $\}$ and any two objects $\mathcal{T}$ and $\mathcal{T}^{\prime}$ in $\mathbf{T T S}_{*}, \mathbf{P}_{*-}$

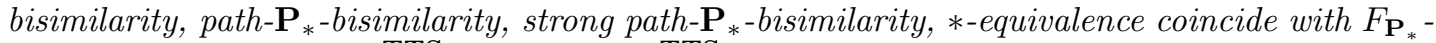
bisimilarity between $\mathcal{B} h_{\mathbf{P}_{*}}^{\mathbf{T T S}}{ }_{*}(\mathcal{T})$ and $\mathcal{B}$ eh $h_{\mathbf{P}_{*}}^{\mathbf{T T S}}{ }_{*}\left(\mathcal{T}^{\prime}\right)$ containing the pair $\left(i_{\mathcal{T}}, i_{\mathcal{T}^{\prime}}\right)$, where $i_{\mathcal{T}}$ : $I_{*} \rightarrow \mathcal{T}$ and $i_{\mathcal{T}^{\prime}}: I_{*} \rightarrow \mathcal{T}^{\prime}{ }^{*}$ are paths, with an initial object $I_{*}$.

\section{References}

[1] P. Aczel and P.F.Mendler. A final coalgebra theorem. In D. H. Pitt and D. E. Rydeheard et al, editors, Proc. of CTCS'89, volume 389 of LNCS, pages 357-365, 1989.

[2] R. Alur, C. Courcoubetis, and T.A. Henzinger. The observational power of clocks. In B. Jonsson and J. Parrow, editors, Proc. of CONCUR'94, volume 836 of LNCS, pages 162-177, 1994.

[3] M.V. Andreeva and I.B. Virbitskaite. Observational timed equivalences for timed stable event structures. Fundamenta Informaticae, 72(4):1-19, 2006.

[4] E. Bihler and W. Vogler. Timed petri nets: Efficiency of asynchronous systems. In M. Bernardo and F. Corradini, editors, Proc. of SFM-RT 2004, volume 3185 of LNCS, pages 25-58, 2004.

[5] G.L. Cattani and V. Sassone. Higher dimentional transition systems. In E. Clarke, editor, Proc. of LICS'96, pages 55-62, New Brunswick, New Jersey, jul 1996.

[6] G. Ciobanu and C. Prisacariu. Refinement of actions and equivalence notions for concurrent systems. Acta Informatica, 37:229-327, 2001. 
[7] G. Ciobanu and C. Prisacariu. Timers for distributed systems. Electronic Notes in Theoretical Computer Science, 164:81-99, 2006.

[8] U. Goltz and H. Wehrheim. Causal testing. In W. Penczek and A. Szalas, editors, Proc. of MFCS'96, volume 1113 of LNCS, pages 394-406, 1996.

[9] N. Gribovskaya and I. Virbitskaite. Timed delay bisimulation is an equivalence relation for timed transition systems. Fundamenta Informaticae, 93(1-3):127-142, 2009.

[10] T.A. Henzinger, R. Majumdar, and V.S. Prabhu. Quantifying similarities between timed systems. In P. Pettersson and W. Yi, editors, Proc. of FORMATS'05, volume 3829 of LNCS, pages 226-241, 2005.

[11] T. Hune and M. Nielsen. Bisimulation and open maps for timed transition systems. Fundamenta Informaticae, 38:61-77, 1999.

[12] B. Jacobs and J. Hughes. Simulations in coalgebra. Theoretical Computer Science, 327(1-2):71108, 2004.

[13] A. Joyal and I. Moerdijk. A completeness theorem for open maps. Annual Pure Applied Logic, 70:51-86, 1997.

[14] A. Joyal, M. Nielsen, and G. Winskel. Bisimulation from open maps. Information and Computation, 127(2):164-185, 1996.

[15] M. Kick, J. Power, and A. Simpson. Coalgebraic semantics for timed processes. Information and Computation, 204(4):588-609, 2006.

[16] S. Lasota. Coalgebra morphisms subsume open maps. Theoretical Computer Science, 280(1):123$135,2002$.

[17] R. Milner and D. Sangiorgi. Barbed bisimulation. In W. Kuich, editor, Proc. of ICALP'92, volume 623 of $L N C S$, pages 685-695, 1992.

[18] L. Monteiro. A coalgebraic characterization of behaviors in the linear time - branching time spectrum. In A. Corradini and U. Montanari, editors, Proc. of WADT'08, volume 5486 of $L N C S$, pages 251-265, 2008.

[19] R. De Nicola and M. Hennessy. Testing equivalence for processes. Theoretical Computer Science, 34:83-133, 1984.

[20] E. Oshevskaya. Open maps bisimulations for higher dimensional automata models. In M. Kutylowski, W. Charatonik, and M. Gebala, editors, Proc. of FCT'09, volume 5699 of LNCS, pages 274-286, 2009.

[21] J. Power and D. Turi. A coalgebraic foundation for linear time semantics. Electronic Notes in Theoretical Computer Science, 29, 1999.

[22] M. Roggenbach and M. Majster-Cederbaum. Towards a unified view of bisimulation: a comparative study. Theoretical Computer Science, 238:81-130, 2000.

[23] J. Rutten. Universal coalgebra: a theory of systems. Theoretical Computer Science, 249(1):3-80, 2000.

[24] B. Steffen and C. Weise. Deciding testing equivalence for real-time processes with dense time. In A. M. Borzyszkowski and S. Sokolowski, editors, Proc. of MFCS'93, volume 711 of $L N C S$, pages 703-713, 1993.

[25] R.J. van Glabbeek. Handbook of process algebras, chapter: The linear time - branching time spectrum I: The Semantics of Concrete, Sequential Processes. Elsevier, 2001.

[26] K. Čerāns. Decidability of bisimulation equivalences for parallel timer processes. In G. von Bochmann and D.K. Probst, editors, Proc. of CAV'92, volume 663 of LNCS, pages 302-315, 1993.

[27] I. Virbitskaite and N. Gribovskaya. Open maps and observational equivalences for timed partial order models. Fundamenta Informaticae, 60(1-4):383-399, 2004.

[28] C. Weise and D. Lenzkes. Efficient scaling-invariant checking of timed bisimulation. In R. Reischuk and M. Morvan, editors, Proc. of STACS'97, volume 1200 of LNCS, pages 176-188, 1997. 